\title{
Cohomology of Kac-Moody groups over a finite field
}

\author{
JAUMe AgUAdÉ
}

ALbERT RUIZ

\begin{abstract}
We compute the mod $p$ cohomology algebra of a family of infinite discrete KacMoody groups of rank two defined over finite fields of characteristic different from $p$.
\end{abstract}

55R35, 81R10, 20G44

\section{Introduction}

A functorial definition of discrete Kac-Moody groups over any commutative ring was established by Tits in 1987 [24] and there is now a great deal of interest in these objects, as illustrated by the recent monograph by Rémy [20] and by some of the references it contains. The work of Kac and Peterson [17] and Kitchloo [18] on the classifying spaces $B K(\mathbb{C})$ of the topological Kac-Moody groups over the complex field were the starting point for the study of these objects from the point of view of the so-called homotopical group theory (see the address by Grodal at the 2010 ICM [15]). Since then, some significant progress has been made in this area. For instance, we know, by Broto and Kitchloo [7], that the mod $p$ cohomology of $B K(\mathbb{C})$ is noetherian. In the case of rank two, the mod $p$ cohomology of $B K(\mathbb{C})$ has been explicitly computed in [4] by Aguadé, Broto, Kitchloo and Saumell, and the self maps of $B K(\mathbb{C})$ have been described and classified by Aguadé and Ruiz in [6].

The main results in this paper (Proposition 8.2 and Theorem 8.3) provide an explicit description of the cohomology algebra $H^{*}\left(B G_{\mathcal{D}}(k) ; \mathbb{F}_{p}\right)$ for $G_{\mathcal{D}}(k)$, a discrete infinite Kac-Moody group of rank two over a finite field $k$ of characteristic different from $p$ (under some restrictions, as stated in Theorem 8.3). To arrive to this result we need detailed descriptions of the root system (Section 3), the parabolic subgroups and the Lévi subgroups (Section 4 ) of $G_{\mathcal{D}}(k)$. The modular invariant theory of the dihedral group plays also a main role in our computation (Sections 6 and 7). Some of these partial steps in the main result may also have interest in their own. Nevertheless, we would like to emphasize that, in spite of the rather technical aspect of some parts of this paper, the path to the computation of the cohomology algebra $H^{*}\left(B G_{\mathcal{D}}(k) ; \mathbb{F}_{p}\right)$ 
is essentially straightforward: First, the problem is reduced to Lévi components (see Proposition 4.3) whose cohomology can be described (Proposition 4.5 to Proposition 4.9 and Proposition 5.1); then, we work out the appropriate kernel and cokernel calculations (see Theorem 7.2) for a Mayer-Vietoris sequence argument.

In the classic case of a finite Weyl group, Friedlander [13] discovered that there is a homotopy equivalence between the $p$-completion of the classifying space of the Chevalley group over the field $\mathbb{F}_{q}$ and the homotopy fixed points of an unstable Adams map $\psi^{q}$ defined on the classifying space of the corresponding compact connected Lie group. These results where extended to $p$-compact groups by Broto and Møller [8] and, recently, to some families of $p$-local compact groups by González [14], obtaining, in both cases, the corresponding $p$-local finite group. In the section of this paper we see that this result does not generalize to the Kac-Moody group case (infinite Weyl group). This suggests a discrepancy between the algebraic and the homotopical definitions of Kac-Moody groups over finite fields, in the case of an infinite Weyl group.

Acknowledgements We want to acknowledge some helpful correspondence and conversations with Alejandro Adem and Bertrand Rémy on some details of our work. The first author is grateful to the Pacific Institute for the Mathematical Sciences in Vancouver for its hospitality during the preparation of this paper. We thank also the referee for his/her accurate reading of our manuscript and for several suggestions that have helped us to improve it.

Remark 1.1 After finishing this paper, we became aware of results in J Foley's PhD thesis [12], having some overlap with the results of this work and, in some points, reaching beyond. This overlap is described in Remarks 4.4 and 9.1.

The authors are partially supported by grants FEDER-MICINN MTM2010-20692 and 2009SGR-1092.

\section{Kac-Moody groups over a field}

In this preliminary section we recall the basic notions and notation of Kac-Moody groups over a field. Our main references are the original paper by Tits [24] and the book by Rémy [20].

A Kac-Moody group functor depends on a set of data $\mathcal{D}$ consisting of a $n \times n$ generalized Cartan matrix $A$, a free abelian group $\Lambda$, and elements $\alpha_{1}, \ldots, \alpha_{n} \in \Lambda$ and $h_{1}, \ldots, h_{n} \in$ $\Lambda^{\vee}=\operatorname{Hom}(\Lambda, \mathbb{Z})$ such that $\left\langle h_{i}, \alpha_{j}\right\rangle=A_{i j}$. From these data $\mathcal{D}$ one can construct a functor $G_{\mathcal{D}}(-)$ from commutative rings to groups which coincides with the classic 
Chevalley-Demazure functor if $A$ is a Cartan matrix. When restricted to fields, the functor $G_{\mathcal{D}}(-)$ can be characterized by a small set of axioms.

Let $k$ be a field and let $k^{+}$and $k^{\times}$be the additive and multiplicative groups of $k$, respectively. The abelian group $T=\operatorname{Hom}\left(\Lambda, k^{\times}\right)$plays the role of a maximal torus in $G_{\mathcal{D}}(k)$ through a monomorphism $\eta: T \rightarrow G_{\mathcal{D}}(k)$. For each $1 \leq i \leq n$ there is a homomorphism $\phi_{i}: \mathrm{SL}_{2}(k) \rightarrow G_{\mathcal{D}}(k)$ as well as two monomorphisms $x_{i}^{+}, x_{i}^{-}: k^{+} \rightarrow G_{\mathcal{D}}(k)$ such that $x_{i}^{+}(r)=\phi_{i}\left(\begin{array}{ll}1 & r \\ 0 & 1\end{array}\right)$ and $x_{i}^{-}(r)=\phi_{i}\left(\begin{array}{cc}1 & 0 \\ -r & 1\end{array}\right)$. The kernel of each $\phi_{i}$ is central in $\mathrm{SL}_{2}(k)$. The group $G_{\mathcal{D}}(k)$ is generated by $T$ and the images of all the $\phi_{i}$. Each $\phi_{i}$ sends diagonal matrices into the maximal torus $T$ as follows: for any $r \in k^{\times}$we have

$$
\phi_{i}\left(\begin{array}{cc}
r & 0 \\
0 & r^{-1}
\end{array}\right)(\lambda)=r^{h_{i}(\lambda)} .
$$

$T$ normalizes $U_{i}^{+}=x_{i}^{+}\left(k^{+}\right)$and $U_{i}^{-}=x_{i}^{-}\left(k^{+}\right)$by the formula

$$
t x_{i}^{ \pm}(r) t^{-1}=x_{i}^{ \pm}\left(t\left(\alpha_{i}\right)^{ \pm 1} r\right)
$$

for any $r \in k$.

The equations $\omega_{i}\left(a_{j}\right)=a_{j}-A_{i j} a_{i}, i, j=1, \ldots, n$ define an action of a group $W$ (the Weyl group) on $\mathbb{Q} a_{1} \oplus \cdots \oplus \mathbb{Q} a_{n}$. The orbit $\Phi=W\left\{a_{1}, \ldots, a_{n}\right\}$ is the root system of $G_{\mathcal{D}}(k)$. Every root is an integral linear combination of $\left\{a_{1}, \ldots, a_{n}\right\}$ with coefficients all positive or all negative. We talk of positive roots $\Phi^{+}$and negative roots $\Phi^{-}$. $W$ is a finite group if and only if the matrix $A$ is a Cartan matrix (ie $A$ is the product of a diagonal matrix and a positive-definite symmetric matrix). The root system depends only on the matrix $A$ and not on the full data $\mathcal{D}$. The Weyl group $W$ acts also on $\Lambda$ (and on $T$ ) by $\omega_{i}(\lambda)=\lambda-\left\langle h_{i}, \lambda\right\rangle \alpha_{i}$, for $\lambda \in \Lambda$ and $i=1, \ldots, n$.

$G_{\mathcal{D}}(k)$ is a group with a double $B N$-pair. There are Borel subgroups $B^{+}, B^{-}$, standard parabolic subgroups $P_{I}^{+}, P_{I}^{-}$for any $I \subset\{1, \ldots, n\}$, root groups $U_{a}$ for any $a \in \Phi$, and all the rich theory of double $B N$-pairs applies.

From a topological point of view, one of the most remarkable properties of Kac-Moody groups with infinite Weyl group, which does not hold in the classic case of a finite Weyl group, is the following.

Theorem 2.1 [19] Let $G$ be a Kac-Moody group with an infinite Weyl group and let $\mathcal{C}$ denote the poset of proper standard parabolic subgroups $P_{I}$ of $G$. Then there is a homotopy equivalence $B G \simeq \operatorname{hocolim}_{\mathcal{C}} B P_{I}$.

The purpose of this paper is to compute $H^{*}\left(B G_{\mathcal{D}}(k) ; \mathbb{F}_{p}\right)$ when the free abelian group $\Lambda$ is of rank two, $k$ is a finite field, the Weyl group of $G_{\mathcal{D}}(k)$ is infinite and $p$ is an odd prime different from the characteristic of the field $k$. 
Throughout this paper we fix the following notation:

(1) $p, \ell$ are different primes, $p$ is odd and $k$ is a finite field of order $q$ and characteristic $\ell$.

(2) $a, b$ are positive integers such that $a b \geq 4$ and $A$ is the generalized Cartan matrix

$$
A=\left(\begin{array}{cc}
2 & -a \\
-b & 2
\end{array}\right)
$$

Without loss of generality, we assume $a \leq b$.

(3) $n_{i}, m_{i}, s_{i}, t_{i}$ for $i=1,2$ are integers such that

$$
\left(\begin{array}{ll}
s_{1} & t_{1} \\
s_{2} & t_{2}
\end{array}\right)\left(\begin{array}{cc}
n_{1} & n_{2} \\
m_{1} & m_{2}
\end{array}\right)=A
$$

We denote $\Delta=s_{1} t_{2}-s_{2} t_{1}, \nabla=n_{1} m_{2}-n_{2} m_{1}$, so that $\Delta \nabla=4-a b$.

(4) $\Lambda$ is a free abelian group of rank two, $\Lambda=\mathbb{Z} e_{1} \oplus \mathbb{Z} e_{2}$, and $\Lambda^{\vee}=\mathbb{Z} e_{1}^{\vee} \oplus \mathbb{Z} e_{2}^{\vee}$ is its $\mathbb{Z}$-dual.

(5) $\alpha_{1}, \alpha_{2} \in \Lambda$ and $h_{1}, h_{2} \in \Lambda^{\vee}$ are the elements $\alpha_{i}=n_{i} e_{1}+m_{i} e_{2}, h_{i}=s_{i} e_{1}^{\vee}+t_{i} e_{2}^{\vee}$, $i=1,2$.

(6) $G$ is the Kac-Moody group $G_{\mathcal{D}}(k)$, where $\mathcal{D}$ consists of the matrix $A$, the lattice $\Lambda$ and the elements $\left\{\alpha_{1}, \alpha_{2}\right\}$ and $\left\{h_{1}, h_{2}\right\}$.

In particular, we want to emphasize that through all this paper the prime $p$ is always assumed to be odd and different from the characteristic of the field $k$.

\section{The root system in rank two}

In this section we will study the root system $\Phi$ associated to the generalized Cartan matrix $A$. Recall that $\Phi$ is the orbit of the basis vectors of $\mathbb{Q}^{2}$ under the action of the infinite dihedral group $W=\left\langle\omega_{1}, \omega_{2}\right\rangle$ acting as

$$
\omega_{1}=\left(\begin{array}{rr}
-1 & a \\
0 & 1
\end{array}\right), \quad \omega_{2}=\left(\begin{array}{rr}
1 & 0 \\
b & -1
\end{array}\right) .
$$

This root system $\Phi$ has been studied in [17], [18], [21] and [23]. The lattice $\Lambda$ and the elements $\alpha_{1}, \alpha_{2} \in \Lambda, h_{1}, h_{2} \in \Lambda^{\vee}$ do not play any role in this section.

If we write $\tau=\omega_{1} \omega_{2}$, then the matrices in $W$ can be described as follows (see [18], [21]):

$$
\tau^{n}=\left(\begin{array}{cc}
d_{2 n+1} & -d_{2 n} \\
c_{2 n} & -c_{2 n-1}
\end{array}\right), \quad \tau^{n} \omega_{1}=\left(\begin{array}{cc}
-d_{2 n+1} & d_{2 n+2} \\
-c_{2 n} & c_{2 n+1}
\end{array}\right),
$$


where the integers $c_{n}, d_{n}$ are defined inductively for any integer $n$ as follows:

$$
\begin{aligned}
c_{0} & =d_{0}=0, & c_{1} & =d_{1}=1, \\
c_{n+1} & =b d_{n}-c_{n-1}, & d_{n+1} & =a c_{n}-d_{n-1} .
\end{aligned}
$$

The following proposition lists many properties of these integers that we will use in this section.

\section{Proposition 3.1}

(1) $c_{2 n+1}=d_{2 n+1}, b d_{2 n}=a c_{2 n}$.

(2) $c_{2 n} \equiv 0(b), c_{2 n+1} \equiv(-1)^{n}(b), d_{2 n} \equiv 0(a), d_{2 n+1} \equiv(-1)^{n}(a)$.

(3) $c_{-n}=-c_{n}, d_{-n}=-d_{n}$.

(4) If $a=1$ and $b=4$, then $d_{2 n}=n, c_{2 n}=4 n, c_{2 n+1}=d_{2 n+1}=2 n+1$.

(5) If $a b>4$, let $\zeta>1$ be a real root of $X^{4}-(a b-2) X^{2}+1$. Then

$$
c_{n}= \begin{cases}\frac{\zeta^{2 n}-1}{\zeta^{n-1}\left(\zeta^{2}-1\right)} & n \text { odd } \\ \frac{b\left(\zeta^{2 n}-1\right)}{\zeta^{n-2}\left(\zeta^{4}-1\right)} & n \text { even }\end{cases}
$$

(6) $c_{n}, d_{n}>0$ for $n>0$.

(7) For $a b>4$ the function $f(n)=c_{2 n+1}-c_{2 n-1}, n>0$, is strictly increasing.

(8) If $a, b>1$ then the sequences $\left\{c_{n}\right\},\left\{d_{n}\right\}$ are strictly increasing.

(9) The sequences $\left\{c_{2 n}\right\},\left\{d_{2 n}\right\},\left\{c_{2 n+1}\right\},\left\{d_{2 n+1}\right\}$ are strictly increasing.

(10) If $(a, b) \neq(1,4)$ then $d_{2 n}<d_{2 n+1}<d_{2 n+4}$ for $n \geq 0$.

(11) If $a=1$ then for $n>1$ we have $d_{2 n+1}<\min \left\{(b-1) d_{2 n},(b-2) d_{2 n-1}\right\}$.

Proof Properties (1)-(6) are either evident or can be easily proven by induction (see [21]).

To prove (7), use (5) to obtain a formula for $f(n)$ and then check that $(d / d x) f(x)>0$ (see Ruiz [21]). To prove (8) by induction notice that if $n$ is odd then

$$
c_{n+1}=b d_{n}-c_{n-1}=b c_{n}-c_{n-1} \geq 2 c_{n}-c_{n-1}>c_{n}
$$

and if $n$ is even then

$$
c_{n+1}=b d_{n}-c_{n-1}=a c_{n}-c_{n-1} \geq 2 c_{n}-c_{n-1}>c_{n}
$$

and similarly with $\left\{d_{n}\right\}$. 
To prove (9) it is enough to consider the case $a=1$. If $b=4$, the result follows from (4). If $b \geq 5$ we know from (7) that $\left\{c_{2 n+1}\right\}$ is strictly increasing. Then, $d_{2 n}=\left(c_{2 n+1}+c_{2 n-1}\right) / b$ and $c_{2 n}=b d_{2 n}$ are also strictly increasing.

In (10) we can also assume $a=1$ and $b \geq 5$. If we write $d_{2 n+1}-d_{2 n}$ as a function of $\zeta$ using (5) then we see that $d_{2 n}<d_{2 n+1}$. We have

$$
\zeta^{4}=(b-2) \zeta^{2}-1 \geq 3 \zeta^{2}-1>\zeta^{2}+1 .
$$

Then if $k=2 n+1$ we see that $\zeta^{2 k+2}>\zeta^{2 k}+\zeta^{2 k-2}-1$ and so

$$
\zeta^{2 k+6}-1>\zeta^{2}\left(\zeta^{2 k}-1\right)\left(\zeta^{2}+1\right)
$$

which implies $d_{k+3}>d_{k}$.

If $a=1$ we have

$$
d_{2 n+1}=b d_{2 n}-d_{2 n-1}=(b-1) d_{2 n}-d_{2 n-1}+d_{2 n}
$$

and

$$
d_{2 n}=d_{2 n-1}-d_{2 n-2}<d_{2 n-1}
$$

for $n>1$. This proves the first inequality in (11). To get the second one, observe

$$
\begin{aligned}
d_{2 n+1}=b d_{2 n}-d_{2 n-1} & =(b-1) d_{2 n-1}-b d_{2 n-2} \\
& =(b-2) d_{2 n-1}+b d_{2 n-2}-d_{2 n-3}-b d_{2 n-2} \\
& <(b-2) d_{2 n-1}
\end{aligned}
$$

if $n>1$.

In this section, it is convenient to denote by $\left\{u_{0}, v_{0}\right\}$ the standard basis of $\mathbb{Q}^{2}$. We will use the following notation $(i \geq 0)$ :

$$
\begin{array}{ll}
u_{i}=\tau^{i} u_{0}=\left(d_{2 i+1}, c_{2 i}\right), & v_{i}=\tau^{-i} v_{0}=\left(d_{2 i}, c_{2 i+1}\right), \\
\bar{u}_{i}=\tau^{i} \omega_{1} v_{0}=\left(d_{2 i+2}, c_{2 i+1}\right), & \bar{v}_{i}=\tau^{-i} \omega_{2} u_{0}=\left(d_{2 i+1}, c_{2 i+2}\right) .
\end{array}
$$

It is clear that these are the positive roots $\Phi^{+}$of the infinite root system $\Phi$. Recall, by Tits [24], that a set of roots $\Psi$ is called prenilpotent if there are elements $\omega, \omega^{\prime} \in W$ such that $\omega \Psi \subset \Phi^{+}$and $\omega^{\prime} \Psi \subset \Phi^{-}$. This notion plays a crucial role in the theory of infinite Kac-Moody groups. The remainder of this section is devoted to investigate when does a pair of roots form a prenilpotent set of roots and also when is the sum of two roots again a root. This is essential to understand the structure of the unipotent subgroups of $G$ (Proposition 4.1). 
We write

$$
\mathcal{A}=\left\{u_{i}, \bar{u}_{i} \mid i \geq 0\right\}, \quad \mathcal{B}=\left\{v_{i}, \bar{v}_{i} \mid i \geq 0\right\}
$$

Proposition 3.2 If $e, w \in \mathcal{A}$ (respectively $\mathcal{B}$ ), then the pair $\{e, w\}$ is prenilpotent.

Proof If $e, w \in \mathcal{A}$ and $N>0$ is large enough then we have $\tau^{-N} e, \tau^{-N} w \in \Phi^{-}$. If $e, w \in \mathcal{B}$ then we may use $\tau^{N}$ for $N>0$ large enough.

Proposition 3.3 If $e, w \in \mathcal{A}$ (respectively $\mathcal{B}$ ) and $a>1$, then $e+w \notin \Phi$.

Proof If $e, w \in \mathcal{A}$ and $e+w \in \mathcal{B}$ then for $N>$ large enough $\tau^{N}(e+w) \in \Phi^{-}$while $\tau^{N}(e), \tau^{N}(w) \in \Phi^{+}$. Hence, if $e+w \in \Phi$ then $e+w \in \mathcal{A}$. Moreover, $W$ leaves invariant the quadratic form

$$
Q(X, Y)=b X^{2}+a Y^{2}-a b X Y
$$

and $Q(e)=Q(w)=Q(e+w)$ is impossible. Hence, any relation $e+w \in \Phi$ should

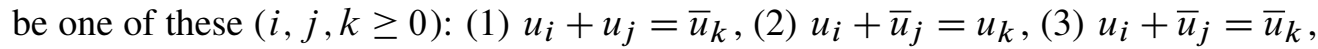
(4) $\bar{u}_{i}+\bar{u}_{j}=u_{k}$.

Each of these equalities can be translated into an equality between coefficients $c_{n}, d_{n}$ as follows:

$$
\begin{aligned}
\left(d_{2 i+1}, c_{2 i}\right)+\left(d_{2 j+1}, c_{2 j}\right) & =\left(d_{2 k+2}, c_{2 k+1}\right), \\
\left(d_{2 i+1}, c_{2 i}\right)+\left(d_{2 j+2}, c_{2 j+1}\right) & =\left(d_{2 k+1}, c_{2 k}\right), \\
\left(d_{2 i+1}, c_{2 i}\right)+\left(d_{2 j+2}, c_{2 j+1}\right) & =\left(d_{2 k+2}, c_{2 k+1}\right), \\
\left(d_{2 i+2}, c_{2 i+1}\right)+\left(d_{2 j+2}, c_{2 j+1}\right) & =\left(d_{2 k+1}, c_{2 k}\right) .
\end{aligned}
$$

In each case, reducing modulo $a$ or $b$ and applying Proposition 3.1(2) we get a contradiction. If we start with $e, w \in \mathcal{B}$ and assume $e+w \in \Phi$, then applying $-\tau^{N}$ for $N$ large enough we obtain a relation $e^{\prime}+w^{\prime} \in \Phi$ with $e^{\prime}, w^{\prime} \in \mathcal{A}$, which we have seen is not possible.

The above result fails for $a=1$ since in this case one sees immediately that

$$
\bar{u}_{0}+\bar{u}_{1}=u_{1}
$$

and applying $-\tau^{-2}$ to this equality,

$$
v_{1}+v_{0}=\bar{v}_{0}
$$

So, applying respectively $\tau^{ \pm i}$, we obtain relations

$$
\bar{u}_{i}+\bar{u}_{i+1}=u_{i+1}, \quad v_{i+1}+v_{i}=\bar{v}_{i} .
$$


Proposition 3.4 If $a=1, b>4, e, w \in \mathcal{A}$ (respectively $\mathcal{B}$ ) and $e+w=f \in \Phi$, then the equality $e+w=f$ is as in (***) above.

Proof Assume $e+w=f \in \Phi$ with $e, w \in \mathcal{A}$, the case of $e, w \in \mathcal{B}$ being equivalent. The same arguments as in Proposition 3.3 imply that $e+w=f$ has to be one of these equalities $(i, j, k \geq 0)$ : (3) $u_{i}+\bar{u}_{j}=\bar{u}_{k}$, (4) $\bar{u}_{i}+\bar{u}_{j}=u_{k}$.

Notice that if $i j k>0$ then $\tau^{-1}$ gives a relation of the same type with smaller subscripts. Hence, we can assume that at least one of the subscripts $i, j, k$ is equal to zero.

Equation (3) implies

$$
d_{2 i+1}+d_{2 j+2}=d_{2 k+2}, \quad c_{2 i}+c_{2 j+1}=c_{2 k+1} .
$$

Hence $d_{2 k+2}>d_{2 j+2}$ and since the sequence $\left\{d_{2 n}\right\}$ is strictly increasing, we get $k>j$. Also, since the sequence $\left\{c_{2 n+1}\right\}$ is strictly increasing, we get $i>0$. Hence, we can assume $j=0$, which amounts to

$$
d_{2 i+1}+1=d_{2 k+2}, \quad c_{2 i}+1=c_{2 k+1} .
$$

Then,

$$
b d_{2 i}-d_{2 i-1}=d_{2 i+1}=d_{2 k+2}-1=d_{2 k+1}-d_{2 k}-1=b d_{2 i}-d_{2 k} .
$$

Using Proposition 3.1(10) we have $d_{2 i+2}>d_{2 i-1}=d_{2 k}<d_{2 k+1}$ and so $i=k$, which is impossible since this would imply $1+d_{2 k+1}=d_{2 k+2}=d_{2 k+1}-d_{2 k}$.

On the other side, equation (4) implies

$$
d_{2 i+2}+d_{2 j+2}=d_{2 k+1}, \quad c_{2 i+1}+c_{2 j+1}=c_{2 k} .
$$

In particular, $k>0$ and without loss of generality we can assume $i=0$. We have

$$
1+d_{2 j+2}=d_{2 k+1}, \quad 1+c_{2 j+1}=c_{2 k},
$$

and then

$$
\begin{aligned}
1+d_{2 j+2} & =d_{2 k+1}=c_{2 k}-d_{2 k-1}=1+d_{2 j+1}-d_{2 k-1}, \\
d_{2 j+2} & =d_{2 j+1}-d_{2 j},
\end{aligned}
$$

and so $d_{2 j}=d_{2 k-1}<d_{2 k+2}$ (using Proposition 3.1(10)) and $j<k+1$ because $\left\{d_{2 n}\right\}$ is strictly increasing. On the other hand, also by Proposition 3.1(10), we have $d_{2 k-2}<d_{2 k-1}=d_{2 j}$ and since $\left\{d_{2 n}\right\}$ is strictly increasing we have $k-1<j$. Hence, $j=k$. Then, $c_{2 k}=1+c_{2 k+1}=1+c_{2 k}-c_{2 k-1}$, which yields $k=1$.

The only remaining case is $a=1, b=4$. In this case, the roots are explicitly computed in Proposition 3.1(4) and the following result can be easily obtained. 
Proposition 3.5 If $a=1, b=4, e, w \in \mathcal{A}$ (respectively $\mathcal{B}$ ) and $e+w=f \in \Phi$, then the equality $e+w=f$ is

$$
\bar{u}_{i}+\bar{u}_{i+2 k-1}=u_{i+k}
$$

for some $i \geq 0, k>0$ (respectively $v_{i}+v_{i+2 k+1}=\bar{v}_{i+k}$ ).

To summarize, we have seen that in the root system $\Phi$ the sum of two positive roots of the same type $(\mathcal{A}$ or $\mathcal{B})$ is almost never a root. The only exceptions are

$$
\begin{aligned}
\bar{u}_{i}+\bar{u}_{i+1} & =u_{i+1}, i \geq 0, & & \text { which occur for } a=1, \\
\bar{u}_{i}+\bar{u}_{i+2 k-1} & =u_{i+k}, i \geq 0, k>0, & & \text { which occur for }(a, b)=(1,4),
\end{aligned}
$$

for type $\mathcal{A}$ and similarly for type $\mathcal{B}$. Our final step in this section is to compute, in each of the cases $e+w \in \Phi$ above, the sets $(\mathbb{N} e+\mathbb{N} w) \cap \Phi$.

Proposition 3.6 If $e, w \in \mathcal{A}$ (respectively $\mathcal{B}$ ) and $e+w \in \Phi$, then

$$
(\mathbb{N} e+\mathbb{N} w) \cap \Phi=\{e+w\} .
$$

Proof We know that we can assume $a=1$. Consider first the case $n \bar{u}_{0}+m \bar{u}_{1}=f \in \Phi$, $n, m>0$. Then, there are only two possibilities: either $f=\bar{u}_{i}$ or $f=u_{i}$. In the first case, we can solve $n \bar{u}_{0}+m \bar{u}_{1}=\bar{u}_{i}$ for $n, m$ and get

$$
\begin{aligned}
n & =(b-1) d_{2 i+2}-(b-2) d_{2 i+1}, \\
m & =d_{2 i+1}-d_{2 i+2} .
\end{aligned}
$$

Then, $n=d_{2 i+1}-(b-1) d_{2 i}$ and then Proposition 3.1(11) yields $n=0$. If $n \bar{u}_{0}+m \bar{u}_{1}=$ $u_{i}$ then we can also solve for $n, m$ :

$$
\begin{aligned}
n & =(b-1) d_{2 i+1}-(b-2) c_{2 i}, \\
m & =c_{2 i}-d_{2 i+1} .
\end{aligned}
$$

Hence, $n=d_{2 i+1}-(b-2) d_{2 i-1}$ and Proposition 3.1(11) yields $n=m=1$.

In the general case of $n \bar{u}_{i}+m \bar{u}_{i+1} \in \Phi$ we can apply $\tau^{-1}$ enough times to get $n \bar{u}_{0}+m \bar{u}_{1} \in \Phi$, which we have already ruled out.

It remains only the case $(a, b)=(1,4)$, where we have to consider

$$
n \bar{u}_{i}+m \bar{u}_{i+2 k-1} \in \Phi, \quad i \geq 0, k>0 .
$$

Using $\tau^{-i}$ we can assume that $i=0$. Recall that the explicit values of $c_{n}$ and $d_{n}$ are given by Proposition 3.1(4). Then, the solutions for $n \bar{u}_{0}+m \bar{u}_{2 k-1}=\bar{u}_{j}$ are

$$
n=1-\frac{j}{2 k-1}, \quad m=\frac{j}{2 k-1},
$$


which are not possible. Also, the solutions for $n \bar{u}_{0}+m \bar{u}_{2 k-1}=u_{j}$ are

$$
n=2-\frac{2 j-1}{2 k-1}, \quad m=\frac{2 j-1}{2 k-1},
$$

which are only possible for $n=m=1$. The proposition is proven.

\section{The parabolic subgroups and the Lévi decomposition}

In the case of the Kac-Moody group $G$ that we are considering, the poset of the proper (positive) parabolic subgroups consists of only three groups $P_{\varnothing}=B^{+}, P_{1}, P_{2}$ and Theorem 2.1 reduces to the fact that $B G$ is the homotopy colimit of the diagram $B P_{1} \leftarrow B P_{\varnothing} \rightarrow B P_{2}$. This is equivalent, by Brown [9, Theorem 7.3], to saying that $G$ is the amalgamated product of $P_{1}$ and $P_{2}$ over $B^{+}$. In this section we want to investigate the group-theoretical structure of these groups.

Like in the case of a finite Weyl group, by Rémy [20, 6.2], there is a Lévi decomposition for parabolic subgroups of Kac-Moody groups $P_{I}=H_{I} \ltimes V_{I}$. In particular, we have that $P_{\varnothing}=B=T \ltimes U^{+}$, where $U^{+}$is the subgroup generated by all positive root groups. The proof of our main theorem requires understanding the structure of the group $U^{+}$. This structure follows from the study of the root system that we have done in Section 3. Using the notation that we introduced there, we have:

Proposition 4.1 [17, Proposition 4.3 and Remark 2] Let $U_{\mathcal{A}}$ (respectively $U_{\mathcal{B}}$ ) be the subgroup generated by all root groups $U_{e}$ for $e \in \mathcal{A}$ (respectively $e \in \mathcal{B}$ ). Denote $E=\bigoplus_{0}^{\infty} k^{+}$. Then:

(1) $U^{+}=U_{\mathcal{A}} * U_{\mathcal{B}}$.

(2) If $a>1$, then both $U_{\mathcal{A}}$ and $U_{\mathcal{B}}$ are isomorphic to $E$.

(3) If $a=1$, then both $U_{\mathcal{A}}$ and $U_{\mathcal{B}}$ are extensions of $E$ by $E$.

Proof (1) is, by Kac and Peterson, in [17, 4.3]. (2) and (3) follow from the analysis in Section 3. If $a>1$ we have seen that the sum of two roots in $\mathcal{A}$ (respectively $\mathcal{B}$ ) is not a root. Hence, $U_{\mathcal{A}}$ and $U_{\mathcal{B}}$ are abelian groups generated by countably many root groups, each one isomorphic to $k^{+}$. If $a=1$ we have seen that in some cases, the sum of two roots in $\mathcal{A}$ can be a root (see Propositions 3.4 and 3.5). However, the subgroup of $U_{\mathcal{A}}$ generated by the root groups $U_{u_{i}}$ for $i \geq 0$ is abelian, isomorphic to $E$ and normal in $U_{\mathcal{A}}$. Also, it follows from Propositions 3.4, 3.5 and 3.6 that the quotient of $U_{\mathcal{A}}$ by this normal subgroup is also abelian and isomorphic to $E$. The same holds for $U_{\mathcal{B}}$. 
To determine the structure of the subgroups $V_{I}$ we need the following lemma.

Lemma 4.2 Let $K$ and $M$ be groups and assume $N$ is a normal subgroup of index $r$ in $K$. Consider the inclusion $N * M<K * M$. Then the normal closure of $N * M$ in $K * M$ is isomorphic to the free product $N *\left(*_{i=1}^{r} M\right)$.

Proof There is an easy topological proof for this lemma. The inclusion $N \triangleleft K$ can be realized topologically by a pointed map of classifying spaces $\pi: B N \rightarrow B K$ which is a regular $r$-fold covering. Let $\left\{x_{1}, \ldots, x_{r}\right\}$ be the fiber of $\pi$ over the base point of $B K$ and consider the map $\pi^{\prime}: \widetilde{X} \rightarrow X$ defined as follows. $X=B K \vee B M$, $\widetilde{X}=B N \vee(B M)_{1} \vee \cdots \vee(B M)_{r}$, where each $(B M)_{i}$ is glued to $B N$ at the point $x_{i}$ and the map $\pi^{\prime}$ is defined so that $\left.\pi^{\prime}\right|_{B N}=\pi$ and $\left.\pi^{\prime}\right|_{(B M)_{i}}=\mathrm{id}$.

Then, $\pi^{\prime}$ is clearly also a regular covering and $\pi^{\prime *}$ identifies $\pi_{1}(\widetilde{X}) \cong N *\left(*_{i=1}^{r} M\right)$ to a normal subgroup of $K * M$. Hence, the normal closure of $N * M$ in $K * M$ is contained in $\pi_{1}(\widetilde{X})$.

On the other side, if $\gamma_{i}$ is a path in $B N$ from the base point to the point $x_{i}$, then the copy of $M$ in $*_{i=1}^{r} M$ corresponding to the summand $(B M)_{i}$ is sent by $\pi^{\prime *}$ to the conjugate $\left[\pi \gamma_{i}\right]^{-1} M\left[\pi \gamma_{i}\right]$ in $K * M$. Hence, $\pi_{1}(\widetilde{X})$ is contained in the normal closure of $N * M$ in $K * M$.

We are now ready to prove that for our cohomology computations we can replace the parabolic subgroups $P_{I}$ by the Lévi subgroups $H_{I}$.

Proposition 4.3 For each $I \varsubsetneqq\{1,2\}$, the homomorphism $P_{I} \rightarrow H_{I}$ induces an isomorphism in cohomology with coefficients in $\mathbb{F}_{p}$.

Proof $P_{I}$ is an extension of $H_{I}$ by $V_{I}$, hence it is enough to prove that the groups $V_{I}$ are $p$-acyclic. Recall that we are assuming that $p$ is prime to the order of $k$ and so the group $k^{+}$is $p$-acyclic. For $I=\varnothing$ we have $V_{\varnothing}=U^{+}$and Proposition 4.1 shows that $U^{+}$is indeed $p$-acyclic. Consider $V_{1}$. According to Rémy [20,6.2] $V_{1}$ is the normal closure in $U^{+}$of the subgroup generated by the root groups $U_{e}$ for $e \in \Phi^{+}-\left\{u_{0}\right\}$. Proposition 4.1 and Lemma 4.2 allow us to compute this normal closure and we see that it is also mod $p$ acyclic.

Remark 4.4 The mod $p$ triviality of the unipotent subgroups of Kac-Moody groups over finite fields of characteristic different from $p$ has been proved independently by Foley in [12]. 
This last result implies that if we take coefficients in $\mathbb{F}_{p}$, then the cohomology of $G$ is isomorphic to the cohomology of the group $\mathrm{H}_{1} *_{T} \mathrm{H}_{2}$. Notice that the hypothesis $p \neq \ell$ is crucial.

The remainder of this section is devoted to study the structure of the groups $H_{i}=$ $\left\langle T, U_{i}^{+}, U_{i}^{-}\right\rangle=\left\langle T, \phi_{i}\left(\mathrm{SL}_{2}(k)\right)\right\rangle$ as well as the homomorphism $\eta: T \rightarrow H_{i}$. It is possible that the next results are implicitly contained in the literature on Kac-Moody groups, but we prefer to work out all details in a self-contained way. Recall from Section 2 the meaning of the integers $n_{i}, m_{i}, s_{i}, t_{i}$ for $i=1,2$. Recall also the action of the Weyl group $W$ on $\Lambda$ given by $\omega_{i}(\lambda)=\lambda-\left\langle h_{i}, \lambda\right\rangle \alpha_{i}$, for $\lambda \in \Lambda$. Since the analysis for $H_{1}$ is the same as for $H_{2}$, we omit all subscripts $i=1,2$ and we write $H, n, m, s, t, \omega$ to simplify the typography.

The homomorphism $\phi: \mathrm{SL}_{2}(k) \rightarrow H$ can have a nontrivial central kernel. We say that $H$ is monic if $\phi$ is injective. The property (*) in Section 2 shows that $H$ is monic if and only if $\ell=2$ or $s, t$ are relatively prime. Notice also that $\operatorname{gcd}(s, t) \in\{1,2\}$.

The proof of the following proposition is straightforward.

Proposition 4.5 Assume $H$ is monic and consider $\psi: k^{\times} \rightarrow k^{\times} \times k^{\times}$given by $\psi(\zeta)=\left(\zeta^{s}, \zeta^{t}\right)$. Then, there is a split exact sequence of abelian groups

$$
k^{\times} \stackrel{\psi}{\longrightarrow} k^{\times} \times k^{\times} \stackrel{\pi}{\longrightarrow} k^{\times}
$$

with $\pi(\zeta, \tau)=\zeta^{-t} \tau^{s}$. If $s, t$ are relatively prime, a section is given by $\sigma(\zeta)=\left(\zeta^{-\mu}, \zeta^{\lambda}\right)$, where $\lambda, \mu$ are integers such that $\lambda s+\mu t=1$. If $s, t$ are both even, then $\ell=2$ and a section is given by $\sigma(\zeta)=\left(\left(\zeta^{1 / 2}\right)^{-m},\left(\zeta^{1 / 2}\right)^{n}\right)$.

Proposition 4.6 Let $E=\mathrm{SL}_{2}(k) \rtimes k^{\times}$with action given by

$$
\zeta \cdot\left(\begin{array}{cc}
x & y \\
z & t
\end{array}\right)=\left(\begin{array}{cc}
x & \zeta^{r} y \\
\zeta^{-r} z & t
\end{array}\right)
$$

for some integer $r$. Then, $E \cong \mathrm{GL}_{2}(k)$ if $r$ is odd and $E \cong \mathrm{SL}_{2}(k) \times k^{\times}$if $r$ is even.

Proof If $r=2 r^{\prime}-1$, consider the homomorphism

$$
(M, \zeta) \longmapsto M\left(\begin{array}{cc}
\zeta^{r^{\prime}} & 0 \\
0 & \zeta^{1-r^{\prime}}
\end{array}\right)
$$

from $\mathrm{SL}_{2}(k) \rtimes k^{\times}$to $\mathrm{GL}_{2}(k)$. If $r=2 r^{\prime}$, consider the homomorphism

$$
(M, \zeta) \longmapsto\left[M\left(\begin{array}{cc}
\zeta^{r^{\prime}} & 0 \\
0 & \zeta^{-r^{\prime}}
\end{array}\right), \zeta\right]
$$

from $\mathrm{SL}_{2}(k) \rtimes k^{\times}$to $\mathrm{SL}_{2}(k) \times k^{\times}$. 
Let $\bar{H}$ denote any of the groups $\mathrm{GL}_{2}(k), \mathrm{SL}_{2}(k) \times k^{\times}$and let $\bar{\eta}$ be the homomorphism $\bar{\eta}: T \rightarrow \bar{H}$ given by

$$
\bar{\eta}(\zeta, \tau)=\left(\begin{array}{ll}
\zeta & 0 \\
0 & \tau
\end{array}\right) \in \mathrm{GL}_{2}(k) \quad \text { and } \quad \bar{\eta}(\zeta, \tau)=\left[\left(\begin{array}{cc}
\zeta & 0 \\
0 & \zeta^{-1}
\end{array}\right), \tau\right] \in \mathrm{SL}_{2}(k) \times k^{\times} .
$$

Let $\bar{\omega}$ be the automorphism of $\Lambda$ given by $\bar{\omega}(e, v)=(v, e)$ if $\bar{H}=\mathrm{GL}_{2}(k)$ and $\bar{\omega}(e, v)=(-e, v)$ if $\bar{H}=\mathrm{SL}_{2}(k) \times k^{\times}$.

We say that $H$ is split if $n, m$ are both even. With this notation, we can state the following structure theorem.

Proposition 4.7 Assume $H$ is monic. Let $\bar{H}=\mathrm{SL}_{2}(k) \times k^{\times}$if $H$ is split and $\bar{H}=\mathrm{GL}_{2}(k)$ if it is not. Then:

(1) There is an isomorphism $\gamma: H \cong \bar{H}$ such that this diagram is commutative:

$$
\begin{gathered}
T \stackrel{\eta}{\longrightarrow} H \\
\left.\gamma\right|_{T} \downarrow \quad \bar{\eta} \cong \downarrow \gamma \\
T \stackrel{\longrightarrow}{\longrightarrow} \bar{H}
\end{gathered}
$$

(2) $\left.\gamma\right|_{T}$ is induced by $M: \Lambda \rightarrow \Lambda$ given by

$$
M= \begin{cases}\left(\begin{array}{cc}
\frac{1}{2} n & -t \\
\frac{1}{2} m & s
\end{array}\right) & \text { if } H \text { is split, } \\
\frac{1}{2}\left(\begin{array}{cc}
n-t & -n-t \\
m+s & -m+s
\end{array}\right) & \text { if } H \text { is not split. }\end{cases}
$$

(3) $M^{-1} \omega M=\bar{\omega}$.

Proof Recall that $H=\left\langle\phi\left(\mathrm{SL}_{2}(k)\right), T\right\rangle$ and $T$ normalizes $\mathrm{SL}_{2}(k)$ according to the formula $(* *)$ in Section 2. Since $H$ is monic we have that $s, t$ are relatively prime and we can apply Proposition 4.5. We have a commutative diagram were each row is an exact sequence and the bottom row is the split exact sequence in Proposition 4.5:

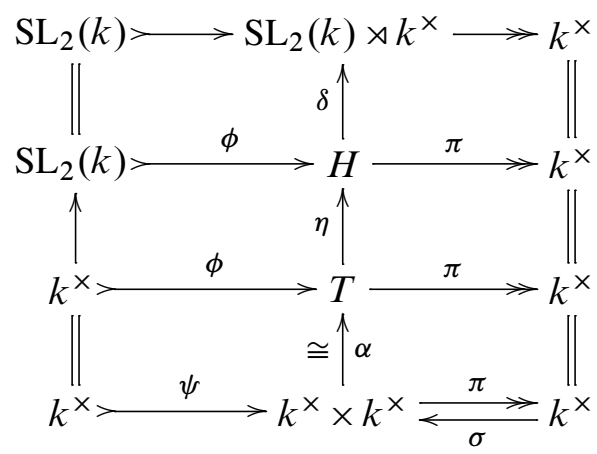


This proves that $H \cong \mathrm{SL}_{2}(k) \rtimes k^{\times}$. To compute the action, choose $\lambda, \mu$ such that $\lambda s+\mu t=1$ and let $r=\lambda m-\mu n$. It is easy to see that $r$ is even if and only if $n, m$ are both even, ie if $H$ is split. Then we conclude that the action of $k^{\times}$on $\mathrm{SL}_{2}(k)$ is given by

$$
\zeta \cdot\left(\begin{array}{ll}
x & y \\
z & t
\end{array}\right)=\left(\begin{array}{cc}
x & \zeta^{r} y \\
\zeta^{-r} z & t
\end{array}\right)
$$

and then Proposition 4.6 proves the first part of this proposition.

To compute the matrix $M$, notice that $\delta$ is given by $\delta(g)=\left(\phi^{-1}\left(g \sigma \pi(g)^{-1}\right), \pi(g)\right)$. Then, some diagram chasing yields

$$
M= \begin{cases}\left(\begin{array}{ll}
\lambda-r^{\prime} t & -\lambda+r^{\prime} t-t \\
\mu+s r^{\prime}-\mu-s r^{\prime}+s
\end{array}\right) & r=2 r^{\prime}-1, H \text { not split, } \\
\left(\begin{array}{cc}
\lambda-r^{\prime} t & -t \\
\mu+r^{\prime} s & s
\end{array}\right) & r=2 r^{\prime}, H \text { split. }\end{cases}
$$

It is easy to see that these matrices coincide with the ones in (2). Knowing these explicit values for $M$, the equality in (3) is immediate.

Consider now the case in which $H$ is not monic. This means that $\ell \neq 2$ and $s=2 s^{\prime}$, $t=2 t^{\prime}$.

Proposition 4.8 Assume $H$ is not monic and consider $\psi: k^{\times} \rightarrow k^{\times} \times k^{\times}$given by $\psi(\zeta)=\left(\zeta^{s}, \zeta^{t}\right)$. Let $d=(q-1) / 2$. There is an exact sequence of abelian groups

$$
k^{\times} /\{ \pm 1\} \stackrel{\psi^{\prime}}{\longrightarrow} k^{\times} \times k^{\times} \stackrel{\pi^{\prime}}{\longrightarrow} k^{\times} \times\{ \pm 1\}
$$

where $\psi^{\prime}$ is induced by $\psi$ and $\pi^{\prime}$ is given by $\pi^{\prime}(\zeta, \tau)=\left(\zeta^{-t^{\prime}} \tau^{s^{\prime}},\left(\zeta^{n} \tau^{m}\right)^{d}\right)$.

Proof Since $H$ is not monic, we have that $q$ is odd and $(s, t)=2$. We know that $n s^{\prime}+m t^{\prime}=1$. The injectivity of $\psi^{\prime}$ as well as the identity $\pi^{\prime} \psi^{\prime}=1$ are clear. Notice that $(\zeta, 1)=\pi^{\prime}\left(\zeta^{-m}, \zeta^{n}\right)$. Also, if $\theta$ is a generator of $k^{\times}$, we have $(1,-1)=\pi^{\prime}\left(\theta^{s^{\prime}}, \theta^{t^{\prime}}\right)$. This proves the surjectivity of $\pi^{\prime}$. Assume $\pi^{\prime}(\zeta, \tau)=(1,1)$. If $n$ is even, then $m$ is odd and this implies that $\tau$ is a square $\tau=\gamma^{2}$. Then, $(\zeta, \tau)=\psi^{\prime}\left(\zeta^{n / 2} \gamma^{m}\right)$. The case $m$ even is similar. Finally, if $n, m$ are both odd, we have $(\zeta, \tau)^{d}=1$ and so $\zeta, \tau$ are both squares or both nonsquares. If $\zeta=\delta^{2}, \tau=\gamma^{2}$, then $(\zeta, \tau)=\psi^{\prime}\left(\delta^{n} \gamma^{m}\right)$. If $\zeta, \tau$ were both nonsquares, the equality $\zeta^{t^{\prime}}=\tau^{s^{\prime}}$ is in contradiction to $n s^{\prime}+m t^{\prime}=1$ with $n, m$ odd. 
Notice that this exact sequence is always split over $k^{\times} \times\{1\}$, a section being given by $\sigma(\zeta, 1)=\left(\zeta^{-m}, \zeta^{n}\right)$. But one sees easily that this exact sequence is not always split over $\{1\} \times\{ \pm 1\}$.

Proposition 4.9 Assume $H$ is not monic. Let $\bar{\eta}: T \rightarrow \mathrm{PGL}_{2}(k) \times k^{\times}$be given by

$$
\bar{\eta}(\zeta, \tau)=\left(\left[\left(\begin{array}{ll}
\zeta & 0 \\
0 & 1
\end{array}\right)\right], \tau\right)
$$

and let $\bar{\omega}: \Lambda \rightarrow \Lambda$ be given by $\bar{\omega}(e, v)=(-e, v)$. Then:

(1) There is an isomorphism $\gamma: H \cong \mathrm{PGL}_{2}(k) \times k^{\times}$such that the following diagram is commutative:

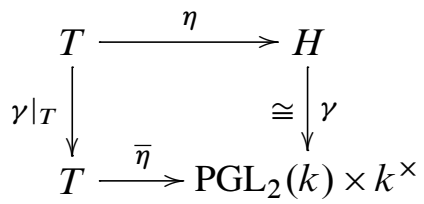

(2) $\left.\gamma\right|_{T}$ is induced by $M: \Lambda \rightarrow \Lambda$ given by

$$
M=\left(\begin{array}{rr}
n & -t / 2 \\
m & s / 2
\end{array}\right) .
$$

(3) $M^{-1} \omega M=\bar{\omega}$.

Proof Since $H$ is not monic, $\phi: \mathrm{SL}_{2}(k) \rightarrow H$ factors through a monomorphism $\phi^{\prime}: \operatorname{PSL}_{2}(k) \rightarrow H$ which fits into a commutative diagram

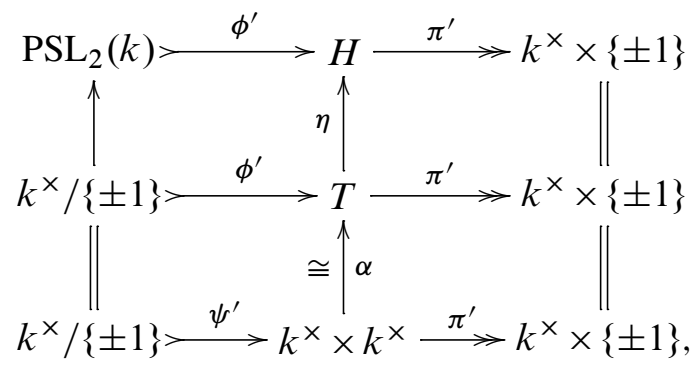

where the bottom row coincides with the exact sequence in Proposition 4.8. This bottom exact sequence is split over $k^{\times} \times\{1\}$ and a section is $\sigma(\zeta, 1)=\left(\zeta^{-m}, \zeta^{n}\right)$. In general, it is not split over $\{1\} \times\{ \pm 1\}$. However, the top exact sequence is split. To see this, consider the element

$$
\beta=\phi\left(\begin{array}{rr}
0 & -1 \\
1 & 0
\end{array}\right) \eta \alpha\left(\theta^{s^{\prime}}, \theta^{t^{\prime}}\right) \in H,
$$

where $\theta \in k^{\times}$is a generator. Then $\pi^{\prime}(\beta)=(1,-1)$ and an easy computation using formulas $(*)$ and $(* *)$ in Section 2 shows that $\beta^{2}=1$. Hence, $H$ is a semidirect product $H \cong \mathrm{PSL}_{2}(k) \rtimes\left(k^{\times} \times\{ \pm 1\}\right)$. 
Then, if we compute the induced action of $k^{\times} \times\{ \pm 1\}$ on $\operatorname{PSL}_{2}(k)$, it turns out that $k^{\times}$acts trivially, while $\{ \pm 1\}$ acts through

$$
\left(\begin{array}{cc}
x & y \\
z & t
\end{array}\right) \mapsto\left(\begin{array}{cc}
t & -\zeta^{-1} z \\
-\zeta y & x
\end{array}\right)
$$

It is easy to identify this extension. Consider the homomorphism $\epsilon: \mathrm{PGL}_{2}(k) \rightarrow\{ \pm 1\}$ which sends to -1 all matrices whose determinant is not a square. The kernel of $\epsilon$ is $\mathrm{PSL}_{2}(k)$ and we have an extension

$$
\operatorname{PSL}_{2}(k) \stackrel{\phi^{\prime}}{\longrightarrow} \operatorname{PGL}_{2}(k) \stackrel{\epsilon}{\longrightarrow}\{ \pm 1\} .
$$

This extension has a section given by

$$
-1 \longmapsto\left(\begin{array}{cc}
0 & -\zeta^{-1} \\
1 & 0
\end{array}\right)
$$

and the action of $\{ \pm 1\}$ on $\operatorname{PSL}_{2}(k)$ is exactly the same as above. This proves that $H \cong \mathrm{PGL}_{2} \times k^{\times}$.

We want to compute now the homomorphism

$$
k^{\times} \times k^{\times} \stackrel{\alpha}{\longrightarrow} T \stackrel{\eta}{\longrightarrow} H \stackrel{\delta}{\longrightarrow} \operatorname{PSL}_{2}(k) \rtimes\left(k^{\times} \times\{ \pm 1\}\right) \longrightarrow \operatorname{PGL}_{2}(k) \times k^{\times} .
$$

We omit the details of this computation which is straightforward once we have an explicit section of the extension and we recall that $\delta$ is given by

$$
\delta(g)=\left(\phi^{\prime-1}\left(g \sigma\left(\pi^{\prime}(g)^{-1}\right)\right), \pi^{\prime}(g)\right) .
$$

Once we have the matrix $M$, the equality in (3) follows immediately.

\section{Cohomology of the parabolic subgroups}

We have seen in Proposition 4.3 that the proper parabolic subgroups $P_{I}$ have the same mod $p$ cohomology as their Lévi factors, which are $T, H_{1}, H_{2}$. We have also seen in Proposition 4.7 and Proposition 4.9 that the groups $H_{1}, H_{2}$ are isomorphic to $\mathrm{GL}_{2}(k)$, $\mathrm{SL}_{2}(k) \times k^{\times}$or $\mathrm{PGL}_{2}(k) \times k^{\times}$. In this section we recollect some known facts about the cohomology of these finite groups. Most of this can be found in Adem and Milgram [1] and Fiedorowicz and Priddy [11]. Recall that we are always assuming that $p$ is an odd prime different from $\ell$.

If $q \not \equiv \pm 1(p)$, then $p$ does not divide the order of these groups and cohomology is trivial in all cases. 
Let $D=k^{\times} \times k^{\times} \cong(\mathbb{Z} /(q-1) \mathbb{Z})^{2}$ be the subgroup of diagonal matrices in $\mathrm{GL}_{2}(k)$. If $q \equiv 1(p)$, then $H^{*}\left(B \mathrm{GL}_{2}(k) ; \mathbb{F}_{p}\right)$ is a ring of invariants, namely

$$
H^{*}\left(B \mathrm{GL}_{2}(k) ; \mathbb{F}_{p}\right) \cong H^{*}\left(B D ; \mathbb{F}_{p}\right)^{C_{2}},
$$

where $C_{2}$ is a cyclic group of order two acting on $D$ by permuting the two factors. Also, if $r$ is such that $q-1=s p^{r}$ with $s$ prime to $p$, then

$$
H^{*}\left(B D ; \mathbb{F}_{p}\right) \cong H^{*}\left(B \mathbb{Z} / p^{r} \mathbb{Z} \times B \mathbb{Z} / p^{r} \mathbb{Z} ; \mathbb{F}_{p}\right)
$$

and

$$
H^{*}\left(B \mathrm{GL}_{2}(k) ; \mathbb{F}_{p}\right) \cong \mathbb{F}_{p}\left[x_{2}, x_{4}\right] \otimes E\left(y_{1}, y_{3}\right)
$$

(the subscripts denote the degrees of the generators) with secondary Bocksteins of height $r$ relating the exterior generators to the polynomial generators.

If $q \equiv-1(p)$, then $H^{*}\left(B D ; \mathbb{F}_{p}\right)$ is trivial but $H^{*}\left(B \mathrm{GL}_{2}(k) ; \mathbb{F}_{p}\right)$ is also a ring of invariants. If $q+1=s p^{r}$ with $s$ prime to $p$, then

$$
H^{*}\left(B \mathrm{GL}_{2}(k) ; \mathbb{F}_{p}\right) \cong H^{*}\left(B \mathbb{Z} / p^{r} \mathbb{Z} ; \mathbb{F}_{p}\right)^{C_{2}} \cong \mathbb{F}_{p}\left[x_{4}\right] \otimes E\left(y_{3}\right),
$$

where $C_{2}$ acts on $\mathbb{Z} / p^{r} \mathbb{Z}$ by multiplication by -1 .

The case of $\mathrm{SL}_{2}(k)=\mathrm{Sp}_{2}(k)$ is similar. If $q \equiv-1(p)$ then

$$
H^{*}\left(B\left(\mathrm{SL}_{2}(k) \times k^{\times}\right) ; \mathbb{F}_{p}\right) \cong H^{*}\left(B \mathrm{SL}_{2}(k) ; \mathbb{F}_{p}\right) \cong H^{*}\left(B \mathrm{GL}_{2}(k) ; \mathbb{F}_{p}\right)
$$

If $q \equiv 1(p)$ then

$$
H^{*}\left(B \mathrm{SL}_{2}(k) ; \mathbb{F}_{p}\right) \cong H^{*}\left(B\left(D \cap \mathrm{SL}_{2}(k)\right) ; \mathbb{F}_{p}\right)^{C_{2}} \cong \mathbb{F}_{p}\left[x_{4}\right] \otimes E\left(y_{3}\right),
$$

where $C_{2}$ acts on $D \cap \mathrm{SL}_{2}(k) \cong \mathbb{Z} /(q-1) \mathbb{Z}$ as multiplication by -1 .

Let us consider now the case of $\operatorname{PGL}_{2}(k)$. If $q \equiv-1(p)$ then $H^{*}\left(B k^{\times} ; \mathbb{F}_{p}\right)$ is trivial and the extension $k^{\times} \longrightarrow \mathrm{GL}_{2}(k) \rightarrow \mathrm{PGL}_{2}(k)$ gives an isomorphism

$$
H^{*}\left(B \mathrm{PGL}_{2}(k) ; \mathbb{F}_{p}\right) \cong H^{*}\left(B \mathrm{GL}_{2}(k) ; \mathbb{F}_{p}\right) \text {. }
$$

On the other side, if $q \equiv 1(p)$ and $r$ is such that $q-1=s p^{r}$ with $s$ prime to $p$, then the Sylow $p$-subgroup of $\mathrm{PGL}_{2}(k)$ is cyclic,

$$
\text { Syl }=\left\{\left[\left(\begin{array}{cc}
x & 0 \\
0 & 1
\end{array}\right)\right] \mid x \in k^{\times}, x^{p^{r}}=1\right\} \cong \mathbb{Z} / p^{r} \mathbb{Z},
$$

and it is easy to compute the stable elements in $H^{*}\left(B S y l ; \mathbb{F}_{p}\right)$. It turns out that the only automorphism of Syl that appears is the involution $x \mapsto x^{-1}$ and so

$$
H^{*}\left(B \mathrm{PGL}_{2}(k) ; \mathbb{F}_{p}\right) \cong H^{*}\left(B \operatorname{Syl} ; \mathbb{F}_{p}\right)^{C_{2}} \cong \mathbb{F}_{p}\left[x_{4}\right] \otimes E\left(y_{3}\right) \text {. }
$$

If we apply all this to the parabolic subgroups $H_{1}, H_{2}$ in $G$, we obtain the following. 
Proposition 5.1 Let $H$ be one of the Lévi subgroups of $G$ and let $\omega \in W$ be the corresponding generating reflection. Then, $H^{*}\left(B H ; \mathbb{F}_{p}\right)$ is trivial unless $q \equiv \pm 1(p)$. If $q \equiv 1(p)$ then $H^{*}\left(B H ; \mathbb{F}_{p}\right) \cong H^{*}\left(B T ; \mathbb{F}_{p}\right)^{\omega}$. If $q \equiv-1(p)$ then $H^{*}\left(B T ; \mathbb{F}_{p}\right)$ is trivial and $H^{*}\left(B H ; \mathbb{F}_{p}\right) \cong \mathbb{F}_{p}\left[x_{4}\right] \otimes E\left(y_{3}\right)$ with a Bockstein relating $y_{3}$ to $x_{4}$. These isomorphism are induced by the inclusion of $T$ in $H$.

Proof This follows from Propositions 4.7 and 4.9 and the discussion above.

In this paper we are assuming that $p$ is an odd prime. It happens that the situation at the prime two is far more complex, even for the Lévi factors. For example, if $p=2$ and $q \equiv 3(4)$, we have $H^{*}\left(B D ; \mathbb{F}_{p}\right) \cong H^{*}\left(B \mathbb{Z} / 2 \mathbb{Z} \times B \mathbb{Z} / 2 \mathbb{Z} ; \mathbb{F}_{2}\right) \cong \mathbb{F}_{2}\left[u_{1}, v_{1}\right]$ and $H^{*}\left(B \mathrm{GL}_{2}(k) ; \mathbb{F}_{2}\right)$ is the subalgebra of $H^{*}\left(B D ; \mathbb{F}_{2}\right)$ generated by the elements

$$
x_{1}=u_{1}+v_{1}, \quad x_{3}=u_{1} v_{1}^{2}+u_{1}^{2} v_{1}, \quad x_{4}=u_{1}^{2} v_{1}^{2},
$$

(see Fiedorowicz and Priddy [11, page 342]). Then, $H^{*}\left(B \mathrm{GL}_{2} ; \mathbb{F}_{2}\right)$ is neither a polynomial ring nor a ring of invariants. Actually,

$$
H^{*}\left(B \mathrm{GL}_{2}(k) ; \mathbb{F}_{2}\right) \cong \mathbb{F}_{2}\left[x_{4}, x_{1}, x_{3}\right] /\left(x_{3}^{2}+x_{4} x_{1}^{2}\right) \text {. }
$$

\section{The Weyl group and its invariants}

The Weyl group $W$ of the Kac-Moody group $G$ is an infinite dihedral group. There are two relevant integral representation of $W$. First, there is the action of $W$ on $\mathbb{Q}^{2}$ given by the root system, as studied in Section 3. On the other side, $W$ acts on the lattice $\Lambda$ by $\omega_{i}(\lambda)=\lambda-\left\langle h_{i}, \lambda\right\rangle \alpha_{i}$ for $i=1,2$. In this section we are interested in the representation given by the action of $W$ on $\Lambda \otimes \mathbb{Z}_{p}$.

The integral $p$-adic representations of an infinite dihedral group were classified by Aguadé, Broto and Saumell in [5] (see also Aguadé [2]) using some explicit invariants which can be easily computed. Let us denote by $v_{p}$ the $p$-adic valuation.

Theorem 6.1 $[5 ; 2]$ Assume $p$ is an odd prime. There are invariants $\Gamma(\rho) \in \mathbb{Z}_{p}$, $\delta_{1}(\rho), \delta_{2}(\rho) \in\{0,1,2, \ldots, \infty\}$ which classify all representations $\rho: W \rightarrow \mathrm{GL}_{2}\left(\mathbb{Z}_{p}\right)$. These invariants can take any value in their range, subject to the relation $\delta_{1}+\delta_{2}=$ $v_{p}(\Gamma)+v_{p}(\Gamma-1)$. If a representation $\rho$ is given by

$$
\omega_{1}=\left(\begin{array}{cc}
1 & 0 \\
0 & -1
\end{array}\right) \text { and } \omega_{2}=M^{-1}\left(\begin{array}{cc}
1 & 0 \\
0 & -1
\end{array}\right) M
$$

with $M=\left(\begin{array}{cc}x & y \\ z & t\end{array}\right)$ (any representation is like that), then

$$
\Gamma(\rho)=x t / \operatorname{det}(M), \quad \delta_{1}(\rho)=v_{p}(x z) \quad \text { and } \quad \delta_{2}(\rho)=v_{p}(y t) .
$$


There is also a similar result for $p=2$ which is more complex and will not be needed in this work (see [5] and [2]).

Let us apply this result to classify the action of $W$ on $\Lambda \otimes \mathbb{Z}_{p}$. Recall from Section 2 the meaning of the integers $a, b, \Delta, \nabla$.

Proposition 6.2 The $p$-adic representation of the Weyl group of $G$ given by the action of $W$ on $\Lambda \otimes \mathbb{Z}_{p}$ is classified by $\Gamma=a b / 4, \delta_{1}=v_{p}(a)+v_{p}(\Delta), \delta_{2}=v_{p}(b)+v_{p}(\nabla)$.

Proof The representation is given by the matrices

$$
\omega_{i}=\left(\begin{array}{rr}
1-s_{i} n_{i} & -t_{i} n_{i} \\
-s_{i} n_{i} & 1-t_{i} n_{i}
\end{array}\right), \quad i=1,2 .
$$

The matrices $P_{i}=\left(\begin{array}{cc}t_{i} & n_{i} \\ -s_{i} & m_{i}\end{array}\right)$ for $i=1,2$ have determinant 2 and satisfy

$$
P_{i}^{-1} \omega_{i} P_{i}=\left(\begin{array}{cc}
1 & 0 \\
0 & -1
\end{array}\right) \text {. }
$$

Hence, the matrix $M$ in Theorem 6.1 is $M=P_{2}^{-1} P_{1}=\frac{1}{2}\left(\begin{array}{cc}-a & \nabla \\ -\Delta & -b\end{array}\right)$.

The action of $W$ on $\Lambda$ gives an action of $W$ on $T=\operatorname{Hom}\left(\Lambda, k^{\times}\right)$and on $H^{1}\left(T ; \mathbb{F}_{p}\right)$. Of course, $H^{1}\left(T ; \mathbb{F}_{p}\right)$ vanishes unless $q \equiv 1(p)$. In this case, $H^{1}\left(T ; \mathbb{F}_{p}\right)=\mathbb{F}_{p}{ }^{2}$ and we have a representation of $W$ in $\mathrm{GL}_{2}\left(\mathbb{F}_{p}\right)$, which is the reduction modulo $p$ of the $p$-adic representation of $W$ studied in Proposition 6.2 and factors through some finite dihedral group $W_{p}$. We are interested in these representations and in their rings of invariants. The mod $p$ reductions of the representations of an infinite dihedral group in $\mathrm{GL}_{2}\left(\mathbb{Z}_{p}\right)$ were studied by Aguadé, Broto, Kitchloo and Saumell (see [4, Table 1]). Let us summarize the results of [4] that we need here. We denote by $P$ a polynomial algebra $P=\mathbb{F}_{p}[x, y]$ with two generators of degree 2 , with the given action of $W_{p}$. There are six types of representations:

- Type I $\Gamma \equiv 0(p), \delta_{1}, \delta_{2}>0 . W_{p}$ is elementary abelian of order 4 and its invariants are $P^{W}=\mathbb{F}_{p}\left[x^{2}, y^{2}\right]$.

- Type II A representation in this type is given, up to the outer automorphism of $W$, by $\Gamma \equiv 0(p), \delta_{1}=0, \delta_{2}>0 . W_{p}$ is a dihedral group of order $4 p$ and its invariants $P^{W_{p}}$ form a polynomial ring on generators $y^{2}, x^{2}\left(x^{p-1}-y^{p-1}\right)^{2}$.

- Type III $W_{p}$ is of order two. $\Gamma \not \equiv 0(p), \delta_{1}, \delta_{2}>0, P^{W}=\mathbb{F}_{p}\left[x, y^{2}\right]$.

- Type IV This type occurs when $\Gamma \not \equiv 0(p), \delta_{1}=0, \delta_{2}>0 . W_{p}$ is then a dihedral group of order $2 p$ in $\mathrm{GL}_{2}\left(\mathbb{F}_{p}\right)$ which is studied in Smith [22, pages 128-129]. The invariants are polynomial in degrees 2 and $4 p$ namely $P^{W}=\mathbb{F}_{p}\left[x,\left(y x^{p-1}-y^{p}\right)^{2}\right]$. 
- Type $\mathrm{V}$ This type occurs when $\Gamma \not \equiv 0(p), \delta_{1}>0, \delta_{2}=0 . W_{p}$ is then a dihedral group of order $2 p$ in $\mathrm{GL}_{2}\left(\mathbb{F}_{p}\right)$ which is also studied in [22, pages 128-129]. The invariants are polynomial in degrees 4 and $2 p$ namely $P^{W}=\mathbb{F}_{p}\left[y^{2}, x\left(x^{p-1}-y^{p-1}\right)\right]$.

- Type VI This type occurs when $\Gamma \not \equiv 0(p), \delta_{1}=\delta_{2}=0$. The order $2 m$ of the dihedral group $W_{p}$ is twice the multiplicative order of the roots (in $\mathbb{F}_{p^{2}}$ ) of the polynomial $X^{2}-2(2 \Gamma-1) X+1$. Thus, $p \equiv \pm 1(m)$. In particular, the order of $W_{p}$ is prime to $p$ and then the invariants $P^{W}$ form a polynomial ring on generators $z_{4}$, $t_{2 m}$ of degrees 4 and $2 m$, respectively. (See [4].)

Let us study in more detail the invariant theory of the representations of type VI. What follows is probably known, but we have not been able to find references for everything that we need in this paper. Hence, this may have some independent interest.

Proposition 6.3 Let $\rho$ be the representation of type VI of the dihedral group of order $2 m$ in $\mathrm{GL}_{2}\left(\mathbb{Z}_{p}\right)$ classified by $\Gamma \in \mathbb{Z}_{p}$. Let $\theta \in \mathbb{F}_{p^{2}}$ be a primitive $m^{\text {th }}$ root of unity. Then, there is a basis $x, y$ such that the ring of invariants $\mathbb{F}_{p}[x, y]^{D_{2 m}}=\mathbb{F}_{p}[f, g]$, where the polynomials $f, g$ can be chosen as follows:

(1) If $p \equiv 1(m)$, then $f=x y, g=x^{m}+y^{m}$.

(2) If $p \equiv-1(m)$, then $f=x^{2}+(2-4 \Gamma) x y+y^{2}$.

(3) If $p \equiv-1(m)$, let $u=\theta x-y, v=\theta y-x$. Then, $g=u^{m}+v^{m}$ if $m$ is even, $m \neq 2$, and $g=\left(u^{m}+v^{m}\right) /\left(\theta-\theta^{-1}\right)$ if $m$ is odd.

Proof If $p \equiv 1(m)$, then $\theta \in \mathbb{F}_{p}$ and the matrices

$$
\omega=\left(\begin{array}{ll}
0 & 1 \\
1 & 0
\end{array}\right), \quad \tau=\left(\begin{array}{cc}
\theta & 0 \\
0 & \theta^{-1}
\end{array}\right),
$$

generate the representation $\rho$. The computation of the invariants of this representation is trivial:

$$
\mathbb{F}_{p}[x, y]^{D_{2 m}}=\mathbb{F}_{p}[f, g], \quad f=x y, g=x^{m}+y^{m} .
$$

If $m=p+1$, then $\theta \notin \mathbb{F}_{p}$, but $\theta^{i}+\theta^{-i}$ and $\left(\theta^{i}-\theta^{-i}\right) /\left(\theta-\theta^{-1}\right)$ are in $\mathbb{F}_{p}$ for all values of $i$ (they are invariant under the Frobenius). Let $\gamma=\theta+\theta^{-1}$. Actually, $\theta, \theta^{-1}$ are the roots of the irreducible polynomial $X^{2}-\gamma X+1$ and so $\theta \in \mathbb{F}_{p^{2}}$. Also, $\gamma=4 \Gamma-2$. Let us consider the matrices

$$
\omega=\left(\begin{array}{ll}
0 & 1 \\
1 & 0
\end{array}\right), \quad \tau=\left(\begin{array}{cc}
\gamma & 1 \\
-1 & 0
\end{array}\right), \quad \omega, \tau \in \mathrm{GL}_{2}\left(\mathbb{F}_{p}\right) .
$$


If we extend scalars to $\mathbb{F}_{p^{2}}$, then the change of basis given by $P=\left(\begin{array}{cc}\theta & -1 \\ -1 & \theta\end{array}\right)$ transforms $\omega, \tau$ into

$$
P^{-1} \omega P=\left(\begin{array}{ll}
0 & 1 \\
1 & 0
\end{array}\right), \quad P^{-1} \tau P=\left(\begin{array}{cc}
\theta & 0 \\
0 & \theta^{-1}
\end{array}\right),
$$

and we see that $\omega, \tau$ generate a dihedral group $D_{2 m} \subset \mathrm{GL}_{2}\left(\mathbb{F}_{p}\right)$ which gives the representation $\rho$. Like before, the computation of the invariants over $\mathbb{F}_{p^{2}}$ is trivial:

$$
\mathbb{F}_{p^{2}}[u, v]^{D_{2 m}}=\mathbb{F}_{p^{2}}[f, g], \quad f=u v, g=u^{p+1}+v^{p+1},
$$

but we are interested in having explicit descriptions of the invariants over $\mathbb{F}_{p}$.

We have $f=u v=-\theta\left(x^{2}-\gamma x y+y^{2}\right)$ and thus $-\theta^{-1} f$ is a degree two invariant in $\mathbb{F}_{p}[x, y]^{D_{2 m}}$. (Notice that $\gamma^{2}-4=\left(\theta-\theta^{-1}\right)^{2}$ is not a square in $\mathbb{F}_{p}$ and so no change of basis can produce a degree two invariant of the form $x y$, in contrast to what is written in [22, page 137].)

Let us consider now the invariant $g(u, v)$. If we write it in the basis $x, y$ we have

$$
g=u^{p+1}+v^{p+1}=\sum_{i=0}^{p+1}(-1)^{i}\left(\begin{array}{c}
p+1 \\
i
\end{array}\right)\left(\theta^{i}+\theta^{-i}\right) x^{i} y^{p-i+1} .
$$

Hence, $g \in \mathbb{F}_{p}[x, y]$ and we have seen that $\mathbb{F}_{p}[x, y]^{D_{2 m}}=\mathbb{F}_{p}\left[\theta^{-1} u v, u^{p+1}+v^{p+1}\right]$.

Consider now the general case $p \equiv-1(m), m \neq 2$. If $m r=p+1$, then $\omega, \tau^{r}$ in $\mathrm{GL}_{2}\left(\mathbb{F}_{p}\right)$ produce the representation $\rho$ of $D_{2 m}$ in $\mathrm{GL}_{2}\left(\mathbb{F}_{p}\right)$ and its invariants over $\mathbb{F}_{p^{2}}$ are generated by $f=u v$ and $g_{m}=u^{m}+v^{m}$. Like before, $x^{2}-\gamma x y+y^{2}$ is an invariant in degree two. The situation with respect to $g_{m}$ is slightly more complicated. If $m$ is even, then

$$
g_{m}=u^{m}+v^{m}=\sum_{i=0}^{m}(-1)^{i}\left(\begin{array}{c}
m \\
i
\end{array}\right)\left(\theta^{i}+\theta^{-i}\right) x^{i} y^{m-i} \in \mathbb{F}_{p}[x, y]
$$

and $\mathbb{F}_{p}[x, y]^{D_{2 m}}=\mathbb{F}_{p}\left[x^{2}-\gamma x y+y^{2}, u^{m}+v^{m}\right]$ like before. On the other side, if $m$ is odd we have

$$
g_{m}=u^{m}+v^{m}=\sum_{i=0}^{m}(-1)^{m-i}\left(\begin{array}{c}
m \\
i
\end{array}\right)\left(\theta^{i}-\theta^{-i}\right) x^{i} y^{m-i} \notin \mathbb{F}_{p}[x, y]
$$

and so $g_{m} /\left(\theta-\theta^{-1}\right) \in \mathbb{F}_{p}[x, y]$. Thus, we have

$$
\mathbb{F}_{p}[x, y]^{D_{2 m}}=\mathbb{F}_{p}\left[x^{2}-\gamma x y+y^{2}, \frac{u^{m}+v^{m}}{\theta-\theta^{-1}}\right] .
$$




\section{The structure of $\operatorname{Coker}(\phi)$}

In this section we will continue the study of the invariant theory of a dihedral group of type VI that we introduced in Section 6. So, $p$ is an odd prime and $D$ is a dihedral group with a representation in $\mathrm{GL}_{2}\left(\mathbb{F}_{p}\right)$ of type VI, classified by $\Gamma \in \mathbb{F}_{p}, \Gamma \neq 0$. The order of $D$ is $2 m$, where $m$ is the order of the roots of $X^{2}-2(2 \Gamma-1) X+1$. Depending on the values of $a, b, \Delta, \nabla$ (see Proposition 6.2), the Weyl group of the Kac-Moody group $G$ acting on $H^{1}\left(T ; \mathbb{F}_{p}\right)$ provides an example of such a representation, but this section is written independently of the theory of Kac-Moody groups.

We use the following notation. $P=\mathbb{F}_{p}\left[v, v^{\prime}\right]$ is a polynomial algebra on two generators of degree two. $S=\mathbb{F}_{p}\left[v, v^{\prime}\right] \otimes E\left(u, u^{\prime}\right)$ is the tensor product of $P$ and an exterior algebra on two generators of degree one. We consider $P$ as a subalgebra of $S$. From a topological point of view, we can think of $P$ and $S$ as $H^{*}\left(B S^{1} \times B S^{1} ; \mathbb{F}_{p}\right)$ and $H^{*}\left(B \mathbb{Z} / p \times B \mathbb{Z} / p ; \mathbb{F}_{p}\right)$, respectively.

Let us consider the following (graded) derivations acting on $S . d$ has degree -1 and is given by

$$
d(u)=d\left(u^{\prime}\right)=0, \quad d(v)=u, \quad d\left(v^{\prime}\right)=u^{\prime} .
$$

$\delta$ has degree +1 and is given by

$$
\delta(v)=\delta\left(v^{\prime}\right)=0, \quad \delta(u)=v, \quad \delta\left(u^{\prime}\right)=v^{\prime} .
$$

Under the identification $S \cong H^{*}\left(B \mathbb{Z} / p \times B \mathbb{Z} / p ; \mathbb{F}_{p}\right), \delta$ corresponds to the Bockstein homomorphism. It is easy to see that if $x \in P$ is a polynomial of degree $2 n$, then $\delta d(x)=n x$.

$D$ acts on the algebras $P$ and $S$ and it is clear that the derivations $d$ and $\delta$ commute with this action. From Proposition 6.3 we know that the ring of invariants of $P$ under $D$ is a polynomial algebra $P^{D}=\mathbb{F}_{p}\left[x_{4}, x_{2 m}\right]$ and we have explicit descriptions of the generators $x_{4}, x_{2 m}$. If we consider the action of $D$ on $S$, [22, Theorem 9.3.2] says that the ring of invariants is

$$
S^{D}=\mathbb{F}_{p}\left[x_{4}, x_{2 m}\right] \otimes E\left(d x_{4}, d x_{2 m}\right) .
$$

$D$ is generated by two elements $\omega_{1}, \omega_{2}$ of order two and we can also consider the rings of invariants of each generating reflection $S^{\omega_{1}}, S^{\omega_{2}}$.

Let $\phi$ be the $P^{D}$ linear map

$$
S^{\omega_{1}} \oplus S^{\omega_{2}} \longrightarrow S
$$

defined by $\phi(t, h)=t+h$. We want to investigate the structure of $\mathcal{S}=\operatorname{Coker}(\phi)$ as a $P^{D}$-module. 
Let us introduce now two relative invariants $\alpha, J$ of $D$ in $S$.

$$
\alpha=u u^{\prime}, \quad J=\left|\begin{array}{cc}
\frac{\partial x_{4}}{\partial v} & \frac{\partial x_{4}}{\partial v^{\prime}} \\
\frac{\partial x_{2 m}}{\partial v} & \frac{\partial x_{2 m}}{\partial v^{\prime}}
\end{array}\right| .
$$

Of course, $J$ is the Jacobian of the algebraically independent polynomials $x_{4}$ and $x_{2 m}$ and so it does not vanish. It is a polynomial of degree $2 m$ in $P$. One sees immediately that both $\alpha$ and $J$ are relative invariants of $D$ with respect to the determinant: for any $g \in D$ we have $g \alpha=\operatorname{det}(g) \alpha$ and $g J=\operatorname{det}(g) J$.

Since the derivations $d$ and $\delta$ commute with the action of $D$ on $S$, we can define two new relative invariants as follows.

$$
\alpha^{\prime}=\delta(\alpha)=v u^{\prime}-v^{\prime} u, \quad J^{\prime}=\frac{1}{m} d(J) .
$$

Notice that $\delta\left(J^{\prime}\right)=J$.

Proposition 7.1 $[\alpha],\left[\alpha^{\prime}\right],[J],\left[J^{\prime}\right]$ are $P^{D}$-linearly independent elements in $\mathcal{S}$.

Proof Let $f\left[\alpha^{\prime}\right]+g[J]=0$ in $\mathcal{S}$ with $f, g \in P^{D}$. This means that there are $p_{1} \in S^{\omega_{1}}$, $p_{2} \in S^{\omega_{2}}$ such that

$$
f \alpha^{\prime}+g J=p_{1}+p_{2} .
$$

Applying $\omega_{1}$ and subtracting we get

$$
2 f \alpha^{\prime}+2 g J=p_{2}-\omega_{1} p_{2} .
$$

Applying $\omega_{2}$ we get

$$
-2 f \alpha^{\prime}-2 g J=p_{2}-\omega_{2} \omega_{1} p_{2} .
$$

Notice that $\alpha^{\prime}$ and $J$ are left invariant by $\omega_{2} \omega_{1}$. Hence, applying $\omega_{2} \omega_{1}$ and adding, we get

$$
-4 f \alpha^{\prime}-4 g J=p_{2}-\left(\omega_{2} \omega_{1}\right)^{2} p_{2} .
$$

Inductively, we get

$$
-2 m f \alpha^{\prime}-2 m g J=p_{2}-\left(\omega_{2} \omega_{1}\right)^{m} p_{2}=0 .
$$

Since $m$ is prime to $p$, this yields $f \alpha^{\prime}+g J=0$ in $S$. But $g J \in P$ and so $f \alpha^{\prime} \in P$ and this can only happen if $f, g=0$.

We have proven that $\left[\alpha^{\prime}\right]$ and $[J]$ are $P^{D}$-linearly independent in $\mathcal{S}$. Consider now a vanishing linear combination $f[\alpha]+g\left[\alpha^{\prime}\right]+h[J]+l\left[J^{\prime}\right]=0$. This means that there are $p_{1} \in S^{\omega_{1}}, p_{2} \in S^{\omega_{2}}$ such that

$$
f \alpha+g \alpha^{\prime}+h J+l J^{\prime}=p_{1}+p_{2} .
$$


If we apply the derivation $\delta$ we get

$$
f \alpha^{\prime}+h J=\delta\left(p_{1}\right)+\delta\left(p_{2}\right)=0
$$

and the argument above yields $f=h=0$. Then, $g \alpha^{\prime}+l J=p_{1}+p_{2}$ and again this implies $g=l=0$.

The following consequence of the proposition above is a key step in our computation of the cohomology of the Kac-Moody group $G$. It displays an interesting parallelism to the computations on $H^{*}(B K(\mathbb{C}))$ in the case of finiteness rank two that appear in Aguadé [3].

Theorem 7.2 $\mathcal{S}$ is a free $P^{D}$-module with basis $\left\{[\alpha],\left[\alpha^{\prime}\right],[J],\left[J^{\prime}\right]\right\}$.

Proof We know that the free $P^{D}$-module with basis $\left\{[\alpha],\left[\alpha^{\prime}\right],[J],\left[J^{\prime}\right]\right\}$ is a submodule of $\mathcal{S}$. It is enough to prove that both graded vector spaces have the same Poincaré series. The kernel of $\phi$ is $S^{D}$. It is very easy to write down the Poincare series of $S$, $S^{\omega_{1}} \oplus S^{\omega_{2}}$ and $S^{D}$. Then, a straightforward computation with power series proves that the Poincare series of $\mathcal{S}$ is as expected.

The computation of the multiplicative structure of $H^{*}\left(B G ; \mathbb{F}_{p}\right)$ in the next section requires that we work out some relations that hold in $S$. Let $y_{3}=d x_{4}, y_{2 m-1}=d x_{2 m}$. Let $\theta, \gamma$ be as in the proof of Proposition 6.3.

Theorem 7.3 The following identities hold in $S$ :

(1) $y_{3} J^{\prime}=m \lambda x_{2 m} \alpha, y_{2 m-1} J^{\prime}=2 m^{2} \mu x_{4}^{m-1} \alpha$,

(2) $y_{3} J=2 x_{4} J^{\prime}-m \lambda x_{2 m} \alpha^{\prime}, y_{2 m-1} J=m x_{2 m} J^{\prime}-2 m^{2} \mu x_{4}^{m-1} \alpha^{\prime}$,

where the coefficients $\lambda$ and $\mu$ are as follows:

(a) $\lambda=1$ if $p \equiv 1(m) ; \lambda=\gamma^{2}-4$ if $p \equiv-1(m)$.

(b) $\quad \mu=1$ if $p \equiv 1(m) ; \mu=\gamma^{2}-4$ if $p \equiv-1(m)$ and $m \neq 2$ is even; $\mu=-1$ if $p \equiv-1(m)$ and $m$ is odd.

Proof The identities in (2) follow from (1) applying the derivation $\delta$. To prove (1) consider

$$
\begin{gathered}
y_{3} J^{\prime}=\frac{1}{m} d x_{4} d J=\frac{1}{m} \mathcal{J}\left(x_{4}, \mathcal{J}\left(x_{4}, x_{2 m}\right)\right) \alpha, \\
y_{2 m-1} J^{\prime}=\frac{1}{m} d x_{2 m} d J=\frac{1}{m} \mathcal{J}\left(x_{2 m}, \mathcal{J}\left(x_{4}, x_{2 m}\right)\right) \alpha,
\end{gathered}
$$


where $\mathcal{J}(f, g)$ denotes the determinant of the Jacobian matrix of the polynomials $f, g$. To compute these double Jacobians, notice that the Jacobian is a relative invariant of the determinant and so if we change bases then the Jacobian is multiplied by the determinant of the change of basis matrix.

We will use the descriptions of $x_{4}$ and $x_{2 m}$ as given in Proposition 6.3.

If $p \equiv 1(m)$, then $x_{4}=v v^{\prime}, x_{2 m}=v^{m}+\left(v^{\prime}\right)^{m}$. Then, a trivial direct calculation gives

$$
\begin{aligned}
\mathcal{J}\left(x_{4}, \mathcal{J}\left(x_{4}, x_{2 m}\right)\right) & =m^{2} x_{2 m}, \\
\mathcal{J}\left(x_{2 m}, \mathcal{J}\left(x_{4}, x_{2 m}\right)\right) & =2 m^{3} x_{4}^{m-1} .
\end{aligned}
$$

If $p \equiv-1(m)$, we extend scalars as to have the $m^{\text {th }}$ root of unity $\theta$ in our field of coefficients. Then $x_{4}$ and $x_{2 m}$ have simple descriptions in a new base $w, w^{\prime}$ which is related to the basis $v, v^{\prime}$ through a matrix $P$ of determinant $\theta^{2}-1$ (see the proof of Proposition 6.3).

If $p \equiv-1(m)$ and $m$ is even, $m \neq 2$, then $x_{4}=-\theta^{-1} w w^{\prime}$ and $x_{2 m}=w^{m}+\left(w^{\prime}\right)^{m}$. In this new basis, the computation of the double Jacobians is as easy as in the case $p \equiv 1(m)$. We get

$$
\begin{aligned}
\mathcal{J}\left(x_{4}, \mathcal{J}\left(x_{4}, x_{2 m}\right)\right) & =\theta^{-2}\left(\theta^{2}-1\right)^{2} m^{2} x_{2 m}=m^{2}\left(\gamma^{2}-4\right) x_{2 m}, \\
\mathcal{J}\left(x_{2 m}, \mathcal{J}\left(x_{4}, x_{2 m}\right)\right) & =-\theta^{-1}\left(\theta^{2}-1\right)^{2} 2 m^{3}\left(w w^{\prime}\right)^{m-1}=2 m^{3}\left(\gamma^{2}-4\right) x_{4}^{m-1} .
\end{aligned}
$$

If $p \equiv-1(m)$ and $m$ is odd, then $x_{4}=-\theta^{-1} w w^{\prime}$ and $x_{2 m}=\left(w^{m}+\left(w^{\prime}\right)^{m}\right) /\left(\theta-\theta^{-1}\right)$. Then

$$
\begin{aligned}
\mathcal{J}\left(x_{4}, \mathcal{J}\left(x_{4}, x_{2 m}\right)\right) & =\theta^{-2}\left(\theta-\theta^{-1}\right)^{-1}\left(\theta^{2}-1\right)^{2} m^{2}\left(w^{m}+\left(w^{\prime}\right)^{m}\right) \\
& =m^{2}\left(\gamma^{2}-4\right) x_{2 m}, \\
\mathcal{J}\left(x_{2 m}, \mathcal{J}\left(x_{4}, x_{2 m}\right)\right) & =-\theta^{-1}\left(\theta-\theta^{-1}\right)^{-2}\left(\theta^{2}-1\right)^{2} 2 m^{3}\left(w w^{\prime}\right)^{m-1} \\
& =-2 m^{3} x_{4}^{m-1} .
\end{aligned}
$$

\section{The cohomology of $B G$}

The preceding sections have provided all ingredients that we need to compute the cohomology algebra of the Kac-Moody group $G$ with coefficients in the field $\mathbb{F}_{p}$. By Theorem 2.1 we know that $B G$ is homotopy equivalent to the push out of

$$
B P_{1} \longleftarrow B P_{\varnothing} \longrightarrow B P_{2}
$$


By Proposition 4.3 we know that $H^{*}\left(B G ; \mathbb{F}_{p}\right) \cong H^{*}\left(B\left(H_{1} *_{T} H_{2}\right) ; \mathbb{F}_{p}\right)$. We have computed $H_{1}$ and $H_{2}$ in Section 4 and we have computed $H^{*}\left(B H_{i} ; \mathbb{F}_{p}\right)$ in Proposition 5.1. Then, the obvious tool to use to compute $H^{*}\left(B\left(H_{1} *_{T} H_{2}\right) ; \mathbb{F}_{p}\right)$ is the Mayer-Vietoris exact sequence. The following lemma on the behavior of the connecting homomorphism of the Mayer-Vietoris exact sequence will be useful.

\section{Lemma 8.1 Let}

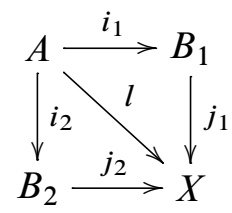

be a push out diagram and let $\Delta: H^{*}(A) \rightarrow H^{*}(X)$ be the connecting homomorphism of the corresponding Mayer-Vietoris exact sequence (coefficient ring omitted). Then, for any $x \in H^{*}(X), t \in H^{*}(A)$, we have $\Delta\left(l^{*}(x) t\right)=x \Delta(t)$.

Proof Let us assume that all maps in the diagram are cofibrations. Then, $\Delta$ is defined through this commutative diagram (see Dold [10, page 49]):

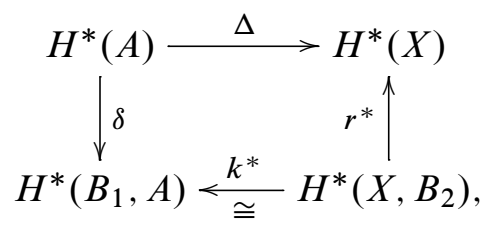

where $r$ and $k$ are the obvious inclusions, $\delta$ is the connecting homomorphism for the couple $\left(B_{1}, A\right)$ and $k$ is an isomorphism because of excision. The way that $\delta$ behaves with respect to cup products (see Dold [10, page 220]) gives

$$
\delta\left(i_{1}^{*}\left(b_{1}\right) t\right)=b_{1} \delta(t), \quad \text { for } b_{1} \in H^{*}\left(B_{1}\right), t \in H^{*}(A) .
$$

Then, an easy calculation using $\Delta=r^{*}\left(k^{*}\right)^{-1} \delta$ gives the formula of the lemma.

Let us deal first with the easy case in which $q \not \equiv 1(p)$. If $\left(A_{1}, \epsilon_{1}\right)$ and $\left(A_{2}, \epsilon_{2}\right)$ are augmented graded $\mathbb{F}_{p}$-algebras, let us denote by $A_{1} \vee A_{2}$ the augmented graded algebra defined as the kernel of $\epsilon_{1}-\epsilon_{2}: A_{1} \oplus A_{2} \rightarrow \mathbb{F}_{p}$.

Proposition 8.2 If $q \not \equiv \pm 1(p)$ then $H^{*}\left(B G ; \mathbb{F}_{p}\right) \cong \mathbb{F}_{p}$. If $q \equiv-1(p)$ then $H^{*}\left(B G ; \mathbb{F}_{p}\right) \cong A \vee A$ with $A=\mathbb{F}_{p}\left[x_{4}\right] \otimes E\left(y_{3}\right)$ (subscripts denote degrees). 
Proof This follows immediately from the Mayer-Vietoris exact sequence $\cdots \longrightarrow H^{*-1}(B T) \stackrel{\Delta}{\longrightarrow} H^{*}(B G) \stackrel{\psi}{\longrightarrow} H^{*}\left(B H_{1}\right) \oplus H^{*}\left(B H_{2}\right) \stackrel{\phi}{\longrightarrow} H^{*}(B T) \longrightarrow \cdots$ and Proposition 5.1.

Of course, the relevant case is when $q \equiv 1(p)$. In this case, the computation of $H^{*}\left(B G ; \mathbb{F}_{p}\right)$ will be made under the following stability hypothesis:

(SH) The prime $p$ is large enough so that $a b(a b-4)$ is prime to $p$.

This hypothesis implies that the representation of the Weyl group $W$ of $G$ is of type VI (see Proposition 6.2). In the geometric language of Aguadé [2], these representations are the ones which have transversally intersecting geodesic paths (see [2, Section 4]).

We use the notation of Section 7. In particular $S^{W}=\mathbb{F}_{p}\left[x_{4}, x_{2 m}\right] \otimes E\left(y_{3}, y_{2 m-1}\right)$ and $P^{W}=\mathbb{F}_{p}\left[x_{4}, x_{2 m}\right]$. We are ready for the main result of this paper:

Theorem 8.3 Assume that $q \equiv 1(p)$ and the stability hypothesis (SH) is satisfied. Then $H^{*}\left(B G ; \mathbb{F}_{p}\right)$ is a $S^{W}$-module with generators $1, \alpha_{3}, \alpha_{4}, J_{2 m}, J_{2 m+1}$ (subscripts denote degrees) and relations

(1) $y_{3} \alpha_{3}=0, y_{2 m-1} \alpha_{3}=0$,

(2) $2 x_{4} \alpha_{3}-y_{3} \alpha_{4}=0, m x_{2 m} \alpha_{3}-y_{2 m-1} \alpha_{4}=0$,

(3) $y_{3} J_{2 m}-m \lambda x_{2 m} \alpha_{3}=0, y_{2 m-1} J_{2 m}-2 m^{2} \mu x_{4}^{m-1} \alpha_{3}=0$,

(4) $y_{3} J_{2 m+1}+m \lambda x_{2 m} \alpha_{4}-2 x_{4} J_{2 m}=0$, $y_{2 m-1} J_{2 m+1}+2 m^{2} \mu x_{4}^{m-1} \alpha_{4}-m x_{2 m} J_{2 m}=0$,

where the parameters $\lambda$ and $\mu$ are as follows:

(1) If $p \equiv 1(m)$, then $\lambda=\mu=1$.

(2) If $p \equiv-1(m)$, then $\lambda=a b(a b-4)$ and $\mu=a b(a b-4)$ if $m \neq 2$ is even and $\mu=-1$ if $m$ is odd.

The algebra structure of $H^{*}\left(B G ; \mathbb{F}_{p}\right)$ is determined by the above relations and the fact that all products between the generators $\alpha_{3}, \alpha_{4}, J_{2 m}, J_{2 m+1}$ vanish. 
Proof As discussed above, we have a Mayer-Vietoris exact sequence (coefficients in $\mathbb{F}_{p}$ are assumed)

$$
\cdots \longrightarrow H^{*-1}(B T) \stackrel{\Delta}{\longrightarrow} H^{*}(B G) \stackrel{\psi}{\longrightarrow} H^{*}\left(B H_{1}\right) \oplus H^{*}\left(B H_{2}\right) \stackrel{\phi}{\longrightarrow} H^{*}(B T) \longrightarrow \cdots
$$

By Proposition 5.1 we know that $H^{*}\left(B H_{i}\right) \cong S^{\omega_{i}}, i=1,2$, where $\omega_{1}, \omega_{2}$ are the generators of the Weyl group $W$. Then, the kernel of $\phi$ is $S^{W}$ and if we denote $\mathcal{S}=\operatorname{Coker}(\phi)$ as in Section 7, we have a short exact sequence

$$
0 \longrightarrow \mathcal{S} \stackrel{\bar{\Delta}}{\longrightarrow} H^{*}(B G) \stackrel{\bar{\psi}}{\longrightarrow} S^{W} \longrightarrow 0 .
$$

Notice that $\bar{\psi}$ is a ring homomorphism and $S^{W}$ is a free graded algebra. Hence, we can choose a section $\sigma$ of $\phi$ which is a ring homomorphism. This section turns $H^{*}(B G)$ into an $S^{W}$-module. Then, Lemma 8.1 implies that $\Delta$ is $S^{W}$-linear. We have that the above short exact sequence is an exact sequence of $P^{W}$-modules. Both $\mathcal{S}$ and $S^{W}$ are free $P^{W}$-modules (Theorem 7.2) and so $H^{*}(B G)$ is a free $P^{W}$-module with basis

$$
\begin{gathered}
1, \quad \sigma\left(y_{3}\right), \quad \sigma\left(y_{2 m-1}\right), \quad \sigma\left(y_{3}\right) \sigma\left(y_{2 m-1}\right), \\
\alpha_{3}=\bar{\Delta}[\alpha], \quad \alpha_{4}=\bar{\Delta}\left[\alpha^{\prime}\right], \quad J_{2 m+1}=\bar{\Delta}[J], \quad J_{2 m}=\bar{\Delta}\left[J^{\prime}\right] .
\end{gathered}
$$

To complete the proof we should compute all products between these generators. First of all, notice that $\Delta$ is a connecting homomorphism which arises from a map $B G \rightarrow \Sigma B T$ and so all products in the image of $\Delta$ vanish. In $S$ we have $y_{3} \alpha=y_{2 m-1} \alpha=0$ and this implies the relations (1) in $H^{*}(B G(k))$. Applying $\delta$ we get the relations (2). Relations (3) and (4) follow from the identities in $S$ proven in Theorem 7.3 and the fact that $\gamma^{2}-4=a b(a b-4)$ in this case.

\section{Comparing $B G$ and the fixed points of an Adams map}

There is a well know result by Friedlander [13] that relates the classifying space of a Chevalley group over a finite field $k$ to the homotopy fixed points of an unstable Adams map from the classifying space of the corresponding compact connected Lie group, after completion at a prime different to the characteristic of $k$. More precisely, let $p, \ell$ be different primes, $k$ a finite field of order $q$ and characteristic $\ell, K$ a compact connected Lie group and $K(q)$ the Chevalley group over $k$ of type $K$. Then, there is 
a homotopy pullback diagram

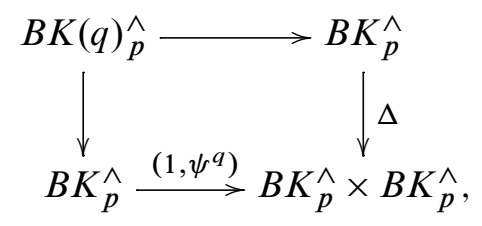

where $\psi^{q}$ is an unstable Adams map of exponent $q$. This result was generalized by Broto and Møller in [8] to the case in which $K$ is a $p$-compact group, and by González [14] to the case where $K$ is in a particular family of $p$-local compact groups. Then, $K(q)$ turns out to be a $p$-local finite group. In this final section we want to investigate if such a pullback diagram could also exist in the context of Kac-Moody groups.

Let $K$ denote the unitary form of a connected, simply connected, not compact KacMoody group as in [16], [18] and [7] and assume that $K$ is of rank two, uniquely determined by two positive integers $a, b$ with $a b>4$. In the case of rank two, there is a satisfactory theory of Adams maps $\psi^{q}: B K \rightarrow B K$ developed in [6] and so, for a prime $p$ and a prime power $q$ with $p \nmid q$, it makes sense to consider the space $X$ defined as the homotopy pullback

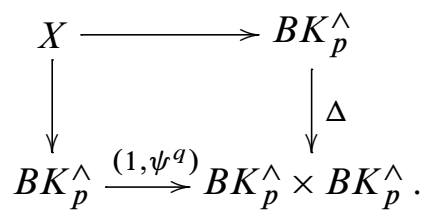

If Friedlander's theorem were true for Kac-Moody groups, then this space $X$ should be homotopy equivalent to $B G_{\mathcal{D}}\left(\mathbb{F}_{q}\right)_{p}^{\wedge}$, where $G_{\mathcal{D}}\left(\mathbb{F}_{q}\right)$ is the corresponding discrete Kac-Moody group. Interestingly enough, this is not true: we will see that, in some particular cases, $X$ and $B G_{\mathcal{D}}\left(\mathbb{F}_{q}\right)_{p}^{\wedge}$ do not have the same mod $p$ cohomology.

Consider $G=G_{\mathcal{D}}\left(\mathbb{F}_{q}\right)$ the discrete Kac-Moody group associated to the data $\mathcal{D}$ consisting of the generalized Cartan matrix $A=\left(\begin{array}{cc}2 & -a \\ -b & 2\end{array}\right)$ and the matrix $\left(\begin{array}{ll}n_{1} & n_{2} \\ m_{1} & m_{2}\end{array}\right)=\left(\begin{array}{ll}1 & 0 \\ 0 & 1\end{array}\right)$ (see Section 2). Let us choose the data $a, b, p, q$ such that the following holds. $p$ and $q$ are odd and relatively prime. $p$ does not divide $a b(a b-4), q \not \equiv \pm 1(p)$ and $q^{m} \equiv 1(p)$, where $m$ is the multiplicative order in $\mathbb{F}_{p^{2}}$ of the roots of $X^{2}+(2-a b) X+1$. It is easy to see that these choices are possible. Under these hypothesis, the mod $p$ cohomology of the classifying space of the (discrete) Kac-Moody group $G$ is trivial (Proposition 8.2). 
The mod $p$ cohomology of the classifying space of the unitary, connected, simply connected, Kac-Moody group $K=K(a, b)$ is computed in [4]:

$$
H^{*}\left(B K ; \mathbb{F}_{p}\right)=\mathbb{F}_{p}\left[x_{4}, y_{2 m}\right] \otimes E\left(z_{2 m+1}\right) .
$$

Let $\psi^{q}: B K \rightarrow B K$ be an unstable Adams map as defined in [6]. When restricted to a maximal torus of $K, \psi^{q}$ is the $q$-power map. Let $X$ be defined by the homotopy pullback above. We will show that the mod $p$ cohomology of $X$ does not vanish in positive degrees.

Consider the Serre spectral sequence in mod $p$ cohomology for the fibration sequence

$$
K \longrightarrow B K \stackrel{\Delta}{\longrightarrow} B K \times B K .
$$

The cohomology of the topological group $K$, by [18], is as follows:

$$
H^{*}\left(K ; \mathbb{F}_{p}\right)=E\left(\tilde{x}_{3}, \tilde{y}_{2 m-1}\right) \otimes \Gamma\left[\tilde{z}_{2 m}\right] .
$$

( $\Gamma$ denotes a divided power algebra.) Then, it is obvious that the elements $\tilde{x}_{3}$ and $\tilde{y}_{2 m-1}$ in the spectral sequence have to be transgressive to $x_{4} \otimes 1-1 \otimes x_{4}$ and $y_{2 m} \otimes 1-1 \otimes y_{2 m}$, respectively. Then, pulling back by $\left(1, \psi^{q}\right)^{*}$ to the Serre spectral sequence for $K \rightarrow X \rightarrow B K$ we see that in this spectral sequence the element $\tilde{y}_{2 m-1}$ is transgressive to $\left(1-q^{m}\right) y_{2 m}=0$ and so it survives to $H^{2 m-1}\left(X ; \mathbb{F}_{p}\right)$.

A similar phenomenon happens also in the case $q \equiv \pm 1(p)$.

This discrepancy between classifying spaces of infinite Kac-Moody groups over finite fields and the homotopy fixed points of the corresponding Adams maps deserves further study.

Remark 9.1 The difference between the cohomology of the classifying space of rank 2 infinite Kac-Moody groups over finite fields and the homotopy fixed points of the corresponding Adams map has been proved independently by Foley in [12].

\section{References}

[1] A Adem, R J Milgram, Cohomology of finite groups, 2nd edition, Grundl. Math. Wissen. 309, Springer, Berlin (2004) MR2035696

[2] J Aguadé, The arboreal approach to pairs of involutions in rank two, Comm. Algebra 37 (2009) 1104-1116 MR2503197

[3] J Aguadé, p-compact groups as subgroups of maximal rank of Kac-Moody groups, J. Math. Kyoto Univ. 49 (2009) 83-112 MR2531131 
[4] J Aguadé, C Broto, N Kitchloo, L Saumell, Cohomology of classifying spaces of central quotients of rank two Kac-Moody groups, J. Math. Kyoto Univ. 45 (2005) 449-488 MR2206359

[5] J Aguadé, C Broto, L Saumell, Rank two integral representations of the infinite dihedral group, Comm. Algebra 35 (2007) 1539-1551 MR2317628

[6] J Aguadé, A Ruiz, Maps between classifying spaces of Kac-Moody groups, Adv. Math. 178 (2003) 66-98 MR1994344

[7] C Broto, N Kitchloo, Classifying spaces of Kac-Moody groups, Math. Z. 240 (2002) 621-649 MR1924024

[8] C Broto, J M Møller, Chevalley p-local finite groups, Algebr. Geom. Topol. 7 (2007) 1809-1919 MR2366180

[9] KS Brown, Cohomology of groups, Graduate Texts in Mathematics 87, Springer, New York (1982) MR672956

[10] A Dold, Lectures on algebraic topology, 2nd edition, Grundl. Math. Wissen. 200, Springer, Berlin (1980) MR606196

[11] Z Fiedorowicz, S Priddy, Homology of classical groups over finite fields and their associated infinite loop spaces, Lecture Notes in Mathematics 674, Springer, Berlin (1978) MR513424

[12] J D Foley, Comparing Kac-Moody groups over the complex numbers and fields of positive characteristic via homotopy theory, $\mathrm{PhD}$ thesis, University of California, San Diego (2012) MR3047264 Available at http://search.proquest.com/ docview/1024147810

[13] E M Friedlander, Computations of $K$-theories of finite fields, Topology 15 (1976) 87-109 MR0394660

[14] A González, Unstable Adams operations acting on p-local compact groups and fixed points, Algebr. Geom. Topol. 12 (2012) 867-908 MR2914621

[15] J Grodal, The classification of p-compact groups and homotopical group theory, from: "Proc. ICM Vol. II", (R Bhatia, A Pal, G Rangarajan, V Srinivas, M Vanninathan, editors), Hindustan Book Agency, New Delhi (2010) 973-1001 MR2827828

[16] V G Kac, Constructing groups associated to infinite-dimensional Lie algebras, from: "Infinite-dimensional groups with applications", (V Kac, editor), Math. Sci. Res. Inst. Publ. 4, Springer, New York (1985) 167-216 MR823320

[17] V G Kac, D H Peterson, Defining relations of certain infinite-dimensional groups, from: "The mathematical heritage of Élie Cartan", Astérisque, SMF, Paris (1985) 165-208 MR837201

[18] N Kitchloo, Topology of Kac-Moody groups, PhD thesis, MIT (1998) Available at http://www.math.jhu.edu/ nitu/papers/Thesis.pdf 
[19] S A Mitchell, Quillen's theorem on buildings and the loops on a symmetric space, Enseign. Math. 34 (1988) 123-166 MR960196

[20] B Rémy, Groupes de Kac-Moody déployés et presque déployés, Astérisque 277, SMF, Paris (2002) viii+348 MR1909671

[21] A Ruiz, Maps between classifying spaces of rank two Kac-Moody groups, $\mathrm{PhD}$ thesis, Universidad Autònoma de Barcelona (2001)

[22] L Smith, Polynomial invariants of finite groups, Research Notes in Mathematics 6, A K Peters Ltd., Wellesley, MA (1995) MR1328644

[23] J Tits, Ensembles ordonnés, immeubles et sommes amalgamées, Bull. Soc. Math. Belg. Sér. A 38 (1986) 367-387 MR885545

[24] J Tits, Uniqueness and presentation of Kac-Moody groups over fields, J. Algebra 105 (1987) 542-573 MR873684

Departament de Matemàtiques, Facultat de Ciències

Universitat Autònoma de Barcelona, 08193 Cerdanyola del Vallès, Spain

Jaume.Aguade@uab.cat, Albert.Ruiz@uab.cat

http://mat.uab.cat/ aguade, http://mat.uab.cat/ albert

Received: 26 June 2012 Revised: 12 March 2013 